\title{
Operators of Gamma White Noise Calculus
}

\author{
Yuri G. Kondratiev \\ Inst. f. Angew. Math., Univ. Bonn, D-53115 Bonn, Germany; and \\ BiBoS, Univ. Bielefeld, D-33615 Bielefeld, Germany; and \\ Inst. Math., NASU, 252601 Kiev, Ukraine
}

Eugene W. Lytvynov

Inst. f. Angew. Math., Univ. Bonn, D-53115 Bonn, Germany; and BiBoS, Univ. Bielefeld, D-33615 Bielefeld, Germany 


\begin{abstract}
The paper is devoted to the study of Gamma white noise analysis. We define an extended Fock space $\mathcal{F}_{\text {Ext }}(\mathcal{H})$ over $\mathcal{H}=L^{2}\left(\mathbb{R}^{d}, d \sigma\right)$, and show how to include the usual Fock space $\mathcal{F}(\mathcal{H})$ in it as a subspace. We introduce in $\mathcal{F}_{\text {Ext }}(\mathcal{H})$ operators $a(\xi)=\int_{\mathbb{R}^{d}} d x \xi(x) a(x)$, $\xi \in S$, with $a(x)=\partial_{x}^{\dagger}+2 \partial_{x}^{\dagger} \partial_{x}+1+\partial_{x}+\partial_{x}^{\dagger} \partial_{x} \partial_{x}$, where $\partial_{x}^{\dagger}$ and $\partial_{x}$ are the creation and annihilation operators at $x$. We show that $(a(\xi))_{\xi \in S}$ is a family of commuting selfadjoint operators in $\mathcal{F}_{\text {Ext }}(\mathcal{H})$ and construct the Fourier transform in generalized joint eigenvectors of this family. This transform is a unitary $I$ between $\mathcal{F}_{\text {Ext }}(\mathcal{H})$ and the $L^{2}$-space $L^{2}\left(S^{\prime}, d \mu_{\mathrm{G}}\right)$, where $\mu_{\mathrm{G}}$ is the measure of Gamma white noise with intensity $\sigma$. The image of $a(\xi)$ under $I$ is the operator of multiplication by $\langle\cdot, \xi\rangle$, so that $a(\xi)$ 's are Gamma field operators. The Fock structure of the Gamma space determined by $I$ coincides with that discovered in [Infinite Dimensional Analysis, Quantum Probability and Related Topics 1 (1998), 91-117]. We note that $I$ extends in a natural way the multiple stochastic integral (chaos) decomposition of the "chaotic" subspace of the Gamma space. Next, we introduce and study spaces of test and generalized functions of Gamma white noise and derive explicit formulas for the action of the creation, neutral, and Gamma annihilation operators on these spaces.
\end{abstract}




\section{Contents}

$\begin{array}{lll}0 & \text { Introduction } & 3\end{array}$

1 Extended Fock space and its rigging 4

2 Gamma field operators $\begin{array}{lr}\text { and the Fourier transform } & 8\end{array}$

3 Chaos decomposition of the Gamma space $\quad 17$

4 Spaces of test and generalized functions and coordinate operators on them 19

5 Standard annihilation operator on Gamma space $\quad 28$

6 Creation, neutral, and Gamma annihilation operators on the Gamma space 


\section{Introduction}

During last years, one witnesses the growth of interest to the the study of compound, or more generally marked Poisson processes and application of these to different problems of probability theory, stochastic analysis and mathematical physics, see e.g. [16, 18]. The Gamma process is an important example of a compound Poisson process with a non-finite measure of the values of jumps of the process in the Lévy formula. In this paper, we derive a white noise calculus for the Gamma process, studying in detail the so-called Gamma field operators. Our interest in the Gamma analysis was inspired, in particular, by the papers by Vershik et al. [34, 35, 33], where the Gamma measure was used in construction of a representation of a group of flows. On the other hand, we improve the results of the paper [22], in which the Fock type structure of the Gamma space was discovered.

Let us shortly describe our results. Let $\sigma$ be a non-atomic Radon measure on $\mathbb{R}^{d}$ and suppose here, for simplicity, that $\operatorname{supp} \sigma=\mathbb{R}^{d}$. In Sect. 1, we introduce the so-called extended Fock space $\mathcal{F}_{\text {Ext }}(\mathcal{H})$ over $\mathcal{H}=L^{2}\left(\mathbb{R}^{d}, d \sigma\right)$. This space is defined as a completion of the set $\mathcal{F}_{\text {fin }}(S)$ with respect to (w.r.t.) a scalar product $(\cdot, \cdot)_{\mathcal{F}_{\text {Ext }}(\mathcal{H})}$, which is defined by using a simple combinatorial rule. Here,

$\mathcal{F}_{\text {fin }}(S):=\bigoplus_{n=0}^{\infty} S_{\mathbb{C}}^{\otimes} n$ is the topological direct sum of the complexified symmetric tensor powers of the Schwartz test space $S$. Thus, $\mathcal{F}_{\text {fin }}(S)$ consists of finite sequences $f=\left(f^{(0)}, f^{(1)}, \ldots, f^{(n)}, 0,0, \ldots\right)$, where $f^{(j)}$ is a smooth symmetric rapidly decreasing function of $j$ variables. We show also the way how to include the usual Fock space over $\mathcal{H}$, denoted by $\mathcal{F}(\mathcal{H})$, into $\mathcal{F}_{\text {Ext }}(\mathcal{H})$, so that $\mathcal{F}(\mathcal{H})$ becomes a proper subspace of $\mathcal{F}_{\text {Ext }}(\mathcal{H})$.

Recall now that the Gamma white noise measure $\mu_{\mathrm{G}}$ with intensity measure $\sigma$ is defined on $S^{\prime}$ - the dual of $S$ w.r.t. the zero space $\mathcal{H}$-by its Laplace transform

$$
\ell_{\mathrm{G}}(\varphi)=\int_{S^{\prime}} \exp [\langle\omega, \varphi\rangle] d \mu_{\mathrm{G}}(\omega)=\exp \left[-\int_{\mathbb{R}^{d}} \log (1-\varphi(x)) d \sigma(x)\right], \quad 1>\varphi \in S
$$

In Sect. 2, we introduce a family of commuting, essentially selfadjoint operators $(a(\xi))_{\xi \in S}$ in $\mathcal{F}_{\text {Ext }}(\mathcal{H})$ with domain $\mathcal{F}_{\text {fin }}(S)$ by the formula

$$
a(\xi)=a^{+}(\xi)+2 a^{0}(\xi)+a^{-}(\xi)+\int_{\mathbb{R}^{d}} \xi d \sigma \cdot \mathrm{id},
$$

where $a^{+}(\xi)$ is a usual creation operator:

$$
a^{+}(\xi) \varphi^{\otimes n}=\xi \widehat{\otimes} \varphi^{\otimes n},
$$

$a^{0}(\xi)$ is a usual neutral operator:

$$
a^{0}(\xi) \varphi^{\otimes n}=n(\xi \varphi) \widehat{\otimes} \varphi^{\otimes(n-1)},
$$


and $a^{-}(\xi)$ is an annihilation operator acting as follows:

$$
a^{-}(\xi) \varphi^{\otimes n}=n\langle\xi, \varphi\rangle \varphi^{\otimes(n-1)}+n(n-1)\left(\xi \varphi^{2}\right) \widehat{\otimes} \varphi^{\otimes(n-2)}
$$

(notice that the first addend corresponds to the usual annihilation operator, while the second addend is an annihilation of a new type).

By using the spectral approach to commutative Jacobi fields in the Fock space $[3,6,24,4]$, we construct the Fourier transform in generalized joint eigenvectors of the family $\left(a^{\sim}(\xi)\right)_{\xi \in S}$, where $a^{\sim}(\xi)$ is the closure of $a(\xi)$. This transform, denoted by $I$, is a unitary between $\mathcal{F}_{\text {Ext }}(\mathcal{H})$ and the Gamma space $L^{2}\left(S^{\prime}, d \mu_{\mathrm{G}}\right):=\left(L_{\mathrm{G}}^{2}\right)$, which was already constructed in [22]. The image of $a^{\sim}(\xi)$ under $I$ is the operator of multiplication by the monomial $\langle\cdot, \xi\rangle$. Thus, $a^{\sim}(\xi)$ 's are actually Gamma field operators.

In Sect. 3, we study the "chaos decomposition" of the Gamma space which naturally appears from its Fock type structure. We show also that the restriction $I \uparrow \mathcal{F}(\mathcal{H})$ of the unitary $I$ to the Fock space as a subspace of $\mathcal{F}_{\text {Ext }}(\mathcal{H})$ is exactly the standard isomorphism between $\mathcal{F}(\mathcal{H})$ and the part of $\left(L_{\mathrm{G}}^{2}\right)$ which appears as a result of the chaos expansion in multiple stochastic integrals w.r.t. the compensated Gamma process. Thus, our approach gives a natural way how, in the Gamma case, to overcome the main difficulty in compound Poisson analysis connected with the fact that these processes do not possess the chaotic representation property.

In Sect. 4, we introduce and study spaces of test and generalized functions of Gamma white noise. This allows us, in particular, to introduce the coordinate operators $\omega(x) \cdot, x \in \mathbb{R}^{d}$, which, as follows from (2), have the form

$$
\omega(x) \cdot=\partial_{x}^{\dagger}+2 \partial_{x}^{\dagger} \partial_{x}+1+\partial_{x}+\partial_{x}^{\dagger} \partial_{x} \partial_{x}
$$

where $\partial_{x}^{\dagger}$ and $\partial_{x}$ are the (images of the) creation and annihilation operators at $x$.

Finally, in Sects. 5 and 6, we derive explicit formulas for the action of the operators $a^{+}(\xi), a^{0}(\xi)$, and $a^{-}(\xi)$. Some formulas appearing here are nothing but infinite-dimensional analogs of the formulas obtained in the one-dimensional case by Meixner in his classical work [29].

\section{Extended Fock space and its rigging}

Let $\sigma$ be a Borel, regular, non-atomic, $\sigma$-finite measure on $\mathbb{R}^{d}, d \in \mathbb{N}$. First, we recall the construction from [25] of a rigging of the real $L^{2}$-space $L^{2}\left(\mathbb{R}^{d}, d \sigma\right)=\mathcal{H}$ by spaces of test and generalized functions of Schwartz type. (Notice that, since $\sigma$ is not necessarily the Lebesgue measure, we need some additional consideration.)

Let $\left(e_{j}\right)_{j=0}^{\infty}$ be the system of Hermite functions on $\mathbb{R}$. For each $p \geq 1$, define $\mathcal{S}_{p}(\mathbb{R})$ to be the real Hilbert space spanned by the orthonormal basis $\left(e_{j}(2 j+2)^{-p}\right)_{j=0}^{\infty}$, and let $\mathcal{S}_{p}\left(\mathbb{R}^{d}\right)=\mathcal{S}_{p}(\mathbb{R})^{\otimes d}$. Considered as a subspace of $L^{2}\left(\mathbb{R}^{d}, d x\right)$, every space 
$\mathcal{S}_{p}\left(\mathbb{R}^{d}\right)$ coincides with the domain of the operator $\left(H^{\otimes d}\right)^{p}$, where $H^{\otimes d}$ is the harmonic oscillator in $L^{2}\left(\mathbb{R}^{d}, d x\right): H^{\otimes d}=-\sum_{i=1}^{d}\left(\frac{d}{d x_{i}}\right)^{2}+\sum_{i=1}^{d} x_{i}^{2}+1$. As well known, $\mathcal{S}\left(\mathbb{R}^{d}\right)=$ proj $\lim _{p \rightarrow \infty} \mathcal{S}_{p}\left(\mathbb{R}^{d}\right)$ is the Schwartz space of rapidly decreasing functions on $\mathbb{R}^{d}$, $\mathcal{S}_{1}\left(\mathbb{R}^{d}\right)$ consists of continuous functions, and

$$
\mathbb{R}^{d} \ni x \mapsto \delta_{x} \in \mathcal{S}_{-1}\left(\mathbb{R}^{d}\right)
$$

is a continuous mapping, where $\mathcal{S}_{-p}\left(\mathbb{R}^{d}\right)$ denotes the dual of $\mathcal{S}_{p}\left(\mathbb{R}^{d}\right)$. We suppose the existence of $\epsilon \geq 0$ such that the space $\mathcal{S}_{1+\epsilon}\left(\mathbb{R}^{d}\right)$ is continuously embedded into $\mathcal{H}$; for example, the following condition holds:

$$
\int_{\mathbb{R}^{d}}\left\|\delta_{x}\right\|_{\mathcal{S}_{-1-\epsilon}\left(\mathbb{R}^{d}\right)}^{2} d \sigma(x)<\infty
$$

Let $O_{p}: S_{p}\left(\mathbb{R}^{d}\right) \hookrightarrow \mathcal{H}$ be an embedding operator. Since $\mathcal{S}\left(\mathbb{R}^{d}\right)$ is a nuclear space, we can suppose without loss of generality that the operator $O_{1+\epsilon}$ is of Hilbert-Schmidt type (in case of the Lebesgue measure, $d \sigma(x)=d x$, we can take $\epsilon=0$ ). Note that, because of the regularity and $\sigma$-finiteness of the measure $\sigma, \mathcal{S}\left(\mathbb{R}^{d}\right)$ is a dense subset of $L^{2}\left(\mathbb{R}^{d}, \sigma\right)$.

Define now $S_{p}$ to be the Hilbert factor space $\mathcal{S}_{p+\epsilon} / \operatorname{ker} O_{p+\epsilon}$. By [5], Ch. 5, Sect. 5, subsec. 1, $\left\{\mathcal{H}, S_{p} \mid p \geq 1\right\}$ is a sequence of compatible Hilbert spaces. Thus, we obtain the rigging

$$
S^{\prime}=\underset{p \rightarrow \infty}{\operatorname{ind} \lim } S_{-p} \supset L^{2}\left(\mathbb{R}^{d}, d \sigma\right)=\mathcal{H} \supset \underset{p \rightarrow \infty}{\operatorname{proj} \lim } S_{p}=S,
$$

where $S_{-p}$, resp. $S^{\prime}$ is the dual of $S_{p}$, resp. $S$ w.r.t. the zero space $\mathcal{H}$. We stress that, for arbitrary $p \geq p^{\prime}$, the space $S_{p}$ is continuously embedded into $S_{p^{\prime}}$ and $|\cdot|_{p} \geq|\cdot|_{p^{\prime}}$, where $|\cdot|_{p}$ denotes the $S_{p}$ norm. Notice that, in fact, the spaces $S_{p}$ and $S$ are completely determined by the support of $\sigma$, i.e., $S$ is the Schwartz test space on $\operatorname{supp} \sigma$.

Now we wish to define an $n$-particle extended Fock space over $\mathcal{H}, \mathcal{F}_{\text {Ext }}^{(n)}(\mathcal{H})$, $n \in \mathbb{N}$. Under a loop $\varkappa$ connecting points $x_{1}, \ldots, x_{m}, m \geq 2$, we understand a class of ordered sets $\left(x_{\pi(1)}, \ldots, x_{\pi(m)}\right)$, where $\pi$ is a permutation of $\{1, \ldots, n\}$, which coincide up to a cyclic permutation. For example, $\left(x_{1}, x_{2}, x_{3}\right)$ and $\left(x_{3}, x_{1}, x_{2}\right)$ define the same loop, while $\left(x_{1}, x_{2}, x_{3}\right)$ and $\left(x_{2}, x_{1}, x_{3}\right)$ define different loops. We put $|\varkappa|=m$. We will interpret also a set $\{x\}$ as a "one-point" loop $\varkappa$, i.e., a loop that comes out of $x,|\varkappa|=1$.

Let $\alpha_{n}=\left\{\varkappa_{1}, \ldots, \varkappa_{\left|\alpha_{n}\right|}\right\}$ be a collection of loops $\varkappa_{j}$ that connect points from the set $\left\{x_{1}, \ldots, x_{n}\right\}$ so that every point $x_{i} \in\left\{x_{1}, \ldots, x_{n}\right\}$ goes into one loop $\varkappa_{j}=\varkappa_{j(i)}$ from $\alpha_{n}$. Here, $\left|\alpha_{n}\right|$ denotes the number of the loops in $\alpha_{n}$, evidently $n=\sum_{j=1}^{\left|\alpha_{n}\right|}\left|\varkappa_{j}\right|$. 
Let $A_{n}$ stand for the set of all possible collections of loops $\alpha_{n}$ over the points $\left\{x_{1}, \ldots, x_{n}\right\}$. Every $\alpha_{n} \in A_{n}$ generates the following continuous mapping

$$
\begin{aligned}
S_{\mathbb{C}}^{\widehat{\otimes} n} \ni f^{(n)}=f^{(n)}( & \left.x_{1}, \ldots, x_{n}\right) \mapsto \\
& \mapsto f_{\alpha_{n}}^{(n)}(\underbrace{x_{1}, \ldots, x_{1}}_{\left|\varkappa_{1}\right| \text { times }}, \underbrace{x_{2}, \ldots, x_{2}}_{\left|\varkappa_{2}\right| \text { times }}, \ldots, \underbrace{x_{\left|\alpha_{n}\right|}, \ldots, x_{\left|\alpha_{n}\right|} \mid}_{\left|\varkappa_{\left|\alpha_{n}\right|}\right| \text { times }}) \in S_{\mathbb{C}}^{\otimes\left|\alpha_{n}\right|},
\end{aligned}
$$

where the lower index $\mathbb{C}$ denotes complexification of a real space and the symbol $\widehat{\otimes}$ stands for the symmetric tensor power. Indeed, for any $p \geq 1$, the diagonalization operator $\mathfrak{D}$ given by

$$
f^{(2)}=f^{(2)}\left(x_{1}, x_{2}\right) \mapsto \mathfrak{D} f^{(2)}=\left(\mathfrak{D} f^{(2)}\right)(x)=f^{(2)}(x, x)
$$

acts continuously from $S_{p, \mathbb{C}}^{\widehat{\otimes} 2}$ into $S_{p, \mathbb{C}}$

$$
\left|\mathfrak{D} f^{(2)}\right|_{p} \leq C_{p}\left|f^{(2)}\right|_{p}
$$

where $|\cdot|_{p}$ denotes also the norm of each space $S_{p, \mathbb{C}}^{\otimes n}, n \in \mathbb{N}$, which yields

$$
\left|f_{\alpha_{n}}^{(n)}\right|_{p} \leq C_{p}^{\left(\left|\varkappa_{1}\right|-1\right)+\left(\left|\varkappa_{2}\right|-1\right)+\cdots+\left(\left|\varkappa_{\mid \alpha_{n}}\right|-1\right)}\left|f^{(n)}\right|_{p},
$$

giving the continuity of the mapping (4).

Thus, we define a scalar product on $S_{\mathbb{C}}^{\widehat{\otimes} n}$ by

$$
\left(f^{(n)}, g^{(n)}\right)_{\mathcal{F}_{\mathrm{Ext}}^{(n)}(\mathcal{H})}=\sum_{\alpha_{n} \in A_{n}} \int_{\mathbb{R}^{d\left|\alpha_{n}\right|}}\left(\overline{f^{(n)}} g^{(n)}\right)_{\alpha_{n}} d \sigma^{\left|\alpha_{n}\right|}
$$

where $\overline{f^{(n)}}$ is the complex conjugate of $f^{(n)}$. Let $\mathcal{F}_{\text {Ext }}^{(n)}(\mathcal{H})$ be the closure of $S_{\mathbb{C}}^{\widehat{\otimes} n}$ in the norm generated by (7).

Remark 1. It is easy to see that the number of the summands in the series (7) is exactly $n$ !. Let us prove this by induction. For $n=1$, this is trivial. Let the statement hold for $n$. At the step $(n+1)$ we add a point $x_{n+1}$ to the set $\left\{x_{1}, \ldots, x_{n}\right\}$. If $x_{n+1}$ goes into a "one-point" loop, then by the supposition there are exactly $n$ ! variants. If $x_{n+1}$ goes into a loop that connect this point with other points from $\left\{x_{1}, \ldots, x_{n}\right\}$, then to each loop connecting $i$ points from $\left\{x_{1}, \ldots, x_{n}\right\}$ we can add the point $x_{n+1}$ in $i$ different ways. That gives $n n$ ! variants. Thus, in total we have exactly $(n+1)$ ! variants.

The extended Fock space $\mathcal{F}_{\text {Ext }}(\mathcal{H})$ over $\mathcal{H}$ is defined as a weighted direct sum of the spaces $\mathcal{F}_{\text {Ext }}^{(n)}(\mathcal{H})$ :

$$
\mathcal{F}_{\text {Ext }}(\mathcal{H})=\bigoplus_{n=0}^{\infty} \mathcal{F}_{\text {Ext }}^{(n)}(\mathcal{H}) n !
$$


where $\mathcal{F}_{\text {Ext }}^{(0)}(\mathcal{H})=\mathbb{C}$ and $0 !=1$. I.e., $\mathcal{F}_{\text {Ext }}(\mathcal{H})$ consists of sequences $f=\left(f^{(0)}, f^{(1)}\right.$, $\left.f^{(2)}, \ldots\right)$ such that $f^{(n)} \in \mathcal{F}_{\text {Ext }}^{(n)}(\mathcal{H})$ and

$$
\|f\|_{\mathcal{F}_{\text {Ext }}(\mathcal{H})}^{2}=\sum_{n=0}^{\infty}\left\|f^{(n)}\right\|_{\mathcal{F}_{\text {Ext }}^{(n)}(\mathcal{H})}^{2} n !<\infty .
$$

Throughout the paper we will identify $f^{(n)} \in \mathcal{F}_{\text {Ext }}^{(n)}(\mathcal{H})$ with the vector

$$
\left(0, \ldots, 0, f^{(n)}, 0,0 \ldots\right) \in \mathcal{F}_{\text {Ext }}(\mathcal{H})
$$

In the following section, we will need a nuclear space that is topologically, i.e., densely and continuously, embedded into $\mathcal{F}_{\text {Ext }}(\mathcal{H})$, and its dual space. Thus, we take

$$
\mathcal{F}_{\text {Ext }}(\mathcal{H}) \supset \mathcal{F}_{\text {fin }}(S), \quad \mathcal{F}_{\text {fin }}^{*}(S) \supset \mathcal{F}_{\text {fin }}(S) .
$$

Here, $\mathcal{F}_{\text {fin }}(S)$ is the topological direct sum of the spaces $S_{\mathbb{C}}^{\widehat{\otimes} n}$, i.e., $\mathcal{F}_{\text {fin }}(S)$ consists of all finite sequences $f=\left(f^{(0)}, f^{(1)}, \ldots, f^{(m)}, 0,0, \ldots\right)$ such that $f^{(n)} \in S_{\mathbb{C}}^{\widehat{\otimes} n}$ and the convergence in $\mathcal{F}_{\text {fin }}(S)$ means the uniform finiteness and the coordinate-wise convergence. That $\mathcal{F}_{\text {fin }}(S)$ is dense in $\mathcal{F}_{\text {Ext }}(\mathcal{H})$ follows directly from the definition of $\mathcal{F}_{\text {Ext }}(\mathcal{H})$. (6), (7), and Remark 1 give

$$
\begin{aligned}
\left\|f^{(n)}\right\|_{\mathcal{F}_{\text {Ext }}^{(n)}(\mathcal{H})}^{2} & \leq n ! \max _{\alpha_{n} \in A_{n}}\left\|f_{\alpha_{n}}^{(n)}\right\|_{\mathcal{H}_{\mathbb{C}}^{\otimes}\left|\alpha_{n}\right|}^{2} \\
& \leq n ! K_{1}^{2 n} \max _{\alpha_{n} \in A_{n}}\left|f_{\left|\alpha_{n}\right|}^{(n)}\right|_{1}^{2} \leq n ! K_{1}^{2 n} C_{1}^{2(n-1)}\left|f^{(n)}\right|_{1}^{2}
\end{aligned}
$$

where $K_{1}$ is the norm of the inclusion operator $S_{1} \hookrightarrow \mathcal{H}$ (we suppose that the constants $\left.K_{1}, C_{1} \geq 1\right)$. (10) implies the continuity of the embedding $\mathcal{F}_{\text {fin }}(S) \hookrightarrow$ $\mathcal{F}_{\text {Ext }}(\mathcal{H})$.

The space $\mathcal{F}_{\text {fin }}^{*}(S)$ in $(9)$ is the dual of $\mathcal{F}_{\text {fin }}(S)$. It will be convenient for us to take it as the dual of $\mathcal{F}_{\text {fin }}(S)$ w.r.t. the zero space $\mathcal{F}(\mathcal{H})$, the usual Fock space over $\mathcal{H}$ :

$$
\mathcal{F}(\mathcal{H})=\bigoplus_{n=0}^{\infty} \mathcal{H}_{\mathbb{C}}^{\widehat{\otimes} n} n !
$$

So, the second inclusion in (9) is part of the nuclear triple (cf. e.g. [5])

$$
\mathcal{F}_{\text {fin }}^{*}(S) \supset \mathcal{F}(\mathcal{H}) \supset \mathcal{F}_{\text {fin }}(S) .
$$

The space $\mathcal{F}_{\text {fin }}^{*}(S)$ consists of infinite sequences $F=\left(F^{(0)}, F^{(1)}, F^{(2)}, \ldots\right)$, where $F^{(n)} \in S_{\mathbb{C}}^{\prime \widehat{\otimes} n}$ and the dualization with $f \in \mathcal{F}_{\text {fin }}(S)$ is given by

$$
\langle\langle F, f\rangle\rangle=\sum_{n=0}^{\infty}\left\langle\overline{F^{(n)}}, f^{(n)}\right\rangle n !,
$$


where $\langle\cdot, \cdot\rangle$ denotes the dualization generated by the scalar product in $\mathcal{H}^{\widehat{\otimes} n}$, which is supposed to be linear in both dots.

Finally, we note that the usual Fock space $\mathcal{F}(\mathcal{H})$ can be included into $\mathcal{F}_{\text {Ext }}(\mathcal{H})$. To this end, we construct a subset of $S_{\mathbb{C}}^{\widehat{\otimes} n}$ of the form

$\mathcal{R}^{(n)}=1$. s. $\left\{\varphi_{1} \widehat{\otimes} \cdots \widehat{\otimes} \varphi_{n} \mid \varphi_{i} \in S_{\mathbb{C}}, \sigma\left(\operatorname{supp} \varphi_{i} \cap \operatorname{supp} \varphi_{j}\right)=0 \forall i, j=1, \ldots, n, i \neq j\right\}$,

where 1. s. denotes the linear span. It follows from $(7)$ that, for arbitrary $f^{(n)}, g^{(n)} \in$ $\mathcal{R}^{(n)}$,

$$
\left(f^{(n)}, g^{(n)}\right)_{\mathcal{F}_{\mathrm{Ext}}^{(n)}(\mathcal{H})}=\left(f^{(n)}, g^{(n)}\right)_{\mathcal{H}_{\mathbb{C}}^{\widehat{\otimes} n}} .
$$

Let $\overline{\mathcal{R}^{(n)}}$ be the closure of $\mathcal{R}^{(n)}$ in the $\mathcal{F}_{\text {Ext }}^{(n)}(\mathcal{H})$ norm. Evidently, $\overline{\mathcal{R}^{(n)}}$ is a subspace of $\mathcal{F}_{\text {Ext }}^{(n)}(\mathcal{H})$. On the other hand, the set $\mathcal{R}^{(n)}$ is dense in $\mathcal{H}_{\mathbb{C}}^{\widehat{\otimes} n}$. Therefore, in view of (12), we can identify $\overline{\mathcal{R}^{(n)}}$ with $\mathcal{H}_{\mathbb{C}}^{\widehat{\otimes} n}$. Then, the subspace $\bigoplus_{n=0}^{\infty} \overline{\mathcal{R}^{(n)}} n$ ! of $\mathcal{F}_{\text {Ext }}(\mathcal{H})$ is identified with $\mathcal{F}(\mathcal{H})$.

Remark 2. Let us explain how the extended Fock space happens to be greater than the usual Fock space. Take, for example, $n=2$. Then, the space $\mathcal{H}_{\mathbb{C}}^{\widehat{\otimes}}=\widehat{L}^{2}\left(\mathbb{R}^{2 d}, d \sigma^{2}\right)$ is the complex space of quadratic integrable symmetric functions of two variables from $\mathbb{R}^{d}$. Of course, any function $f^{(2)}$ from $\mathcal{H}_{\mathbb{C}}^{\widehat{\otimes} 2}$ is defined on $\mathbb{R}^{2 d}$ up to a set of zero $\sigma^{2}$ measure. The diagonal $\left\{(x, x) \mid x \in \mathbb{R}^{d}\right\}$ in $\mathbb{R}^{2 d}$ has $\sigma^{2}$ measure zero, while $\sigma$ is non-atomic. On the other hand, every function $f^{(2)} \in \mathcal{F}_{\text {Ext }}^{(2)}(\mathcal{H})$ must also be defined on the diagonal up to a set of zero $\sigma$ measure, if we identify this diagonal with $\mathbb{R}^{d}$. Thus, any function $f^{(2)} \in \mathcal{H}_{\mathbb{C}}^{\widehat{\otimes} 2}$ is included into $\mathcal{F}_{\text {Ext }}^{(2)}(\mathcal{H})$ if we put additionally that $f^{(2)}$ is equal to zero on the diagonal. An analogous situation takes place in all the other $n$-particle spaces with $n \geq 3$.

Remark 3. Let us stress that we have constructed two different types of inclusion: the first one $\mathcal{F}_{\text {fin }}(S) \subset \mathcal{F}(\mathcal{H})$ in $(11)$ and $\mathcal{F}(\mathcal{H})$ as a subspace of $\mathcal{F}_{\text {Ext }}(\mathcal{H})$.

\section{Gamma field operators and the Fourier transform}

In this section, we will define a family of Gamma field operators and construct a Fourier transform in generalized joint eigenvectors of this family.

For each $\xi \in S$, let $a^{+}(\xi)$ be the standard creation operator defined on $\mathcal{F}_{\text {fin }}(S)$ :

$$
a^{+}(\xi) f^{(n)}=\xi \widehat{\otimes} f^{(n)}, \quad f^{(n)} \in S_{\overparen{C}}^{\widehat{\otimes} n}, n \in \mathbb{N}_{0}=\{0,1,2, \ldots\} .
$$

Because of the estimate

$$
\left|\xi \widehat{\otimes} f^{(n)}\right|_{p} \leq|\xi|_{p}\left|f^{(n)}\right|_{p},
$$


the operator $a^{+}(\xi)$ acts continuously on $\mathcal{F}_{\text {fin }}(S)$.

Let us calculate the adjoint operator of $a^{+}(\xi)$ in $\mathcal{F}_{\text {Ext }}(\mathcal{H})$, which will be denoted by $a^{-}(\xi)$. By (7), (8), and (13), we have for arbitrary $\xi, \varphi, \psi \in S$,

$$
\begin{aligned}
& \left(a^{+}(\xi) \varphi^{\otimes n}, \psi^{\otimes(n+1)}\right)_{\mathcal{F}_{\mathrm{Ext}}(\mathcal{H})}=(n+1) !\left(\xi \widehat{\otimes} \varphi^{\otimes n}, \psi^{\otimes(n+1)}\right)_{\mathcal{F}_{\mathrm{Ext}}^{(n+1)}(\mathcal{H})} \\
& =(n+1) ! \sum_{\alpha_{n+1} \in A_{n+1}} \int_{\mathbb{R}^{d\left|\alpha_{n+1}\right|}}\left((\xi \psi) \widehat{\otimes}(\varphi \psi)^{\otimes n}\right)_{\alpha_{n+1}} d \sigma^{\left|\alpha_{n+1}\right|} \\
& =(n+1) !\left[\langle\xi, \psi\rangle \sum_{\alpha_{n} \in A_{n}} \int_{\mathbb{R}^{d\left|\alpha_{n}\right|}}\left((\varphi \psi)^{\otimes n}\right)_{\alpha_{n}} d \sigma^{\left|\alpha_{n}\right|}\right. \\
& \left.\quad+n \sum_{\alpha_{n} \in A_{n}} \int_{\mathbb{R}^{d\left|\alpha_{n}\right|}}\left(\left(\xi \varphi \psi^{2}\right) \widehat{\otimes}(\varphi \psi)^{\otimes(n-1)}\right)_{\alpha_{n}} d \sigma^{\left|\alpha_{n}\right|}\right] \\
& =\left(\varphi^{\otimes n},(n+1)\langle\xi, \psi\rangle \psi^{\otimes n}+(n+1) n\left(\xi \psi^{2}\right) \widehat{\otimes} \psi^{\otimes(n-1)}\right)_{\mathcal{F}_{\mathrm{Ext}}(\mathcal{H})}
\end{aligned}
$$

where we used just as in Remark 1 the observation that a new point can be added to a loop connecting $i$ points in $i$ different ways. Thus,

$$
a^{-}(\xi)=a_{1}^{-}(\xi)+a_{2}^{-}(\xi)
$$

where $a_{1}^{-}(\xi)$ is the standard annihilation operator:

$$
a_{1}^{-}(\xi) \varphi^{\otimes n}=n\langle\xi, \varphi\rangle \varphi^{\otimes(n-1)}
$$

and $a_{2}^{-}(\xi)$ given by

$$
a_{2}^{-}(\xi) \varphi^{\otimes n}=n(n-1)\left(\xi \varphi^{2}\right) \widehat{\otimes} \varphi^{\otimes(n-2)}
$$

is a "Gamma annihilation" operator, which appears because of the nonstandard scalar product in our Fock space. Due to $(5), a^{-}(\xi)$ acts continuously on $\mathcal{F}_{\text {fin }}(S)$ and

$$
\begin{aligned}
& \left|a_{1}^{-}(\xi) f^{(n)}\right|_{p} \leq n|\xi|_{-p}\left|f^{(n)}\right|_{p}, \\
& \left|a_{2}^{-}(\xi) f^{(n)}\right|_{p} \leq n(n-1) C_{p}^{2}|\xi|_{p}\left|f^{(n)}\right|_{p},
\end{aligned}
$$

Finally, we define on $\mathcal{F}_{\text {fin }}(S)$ the neutral operator $a^{0}(\xi), \xi \in S$, as the differential second quantization of the operator of multiplication by $\xi$ :

$$
a^{0}(\xi) \varphi^{\otimes n}=n(\xi \varphi) \widehat{\otimes} \varphi^{\otimes(n-1)}, \quad \varphi \in S .
$$

Again, $a^{0}(\xi)$ acts continuously on $\mathcal{F}_{\text {fin }}(S)$ and

$$
\left|a^{0}(\xi) f^{(n)}\right|_{p} \leq n C_{p}|\xi|_{p}\left|f^{(n)}\right|_{p}, \quad f^{(n)} \in S_{\mathbb{C}}^{\widehat{\otimes} n}
$$


Thus, we are in position to define, for each $\xi \in S$, the Gamma field operator $a(\xi)$ on $\mathcal{F}_{\text {fin }}(S)$ :

$$
a(\xi)=a^{+}(\xi)+2 a^{0}(\xi)+\langle\xi\rangle \mathrm{id}+a^{-}(\xi),
$$

where $\langle\xi\rangle=\int_{\mathbb{R}^{d}} \xi(x) d \sigma(x)$ and id denotes the identity operator. Each $a(\xi)$ with domain $\mathcal{F}_{\text {fin }}(S)$ is a Hermitian operator in $\mathcal{F}_{\text {Ext }}(\mathcal{H})$.

Lemma 1 The operators $a(\xi), \xi \in S$, with domain $\mathcal{F}_{\text {fin }}(S)$ are essentially selfadjoint in $\mathcal{F}_{\text {Ext }}(\mathcal{H})$ and their closures $a^{\sim}(\xi)$ constitute a family of commuting selfadjoint operators, where the commutation is understood in the sense of the resolutions of the identity.

Proof. Let us show that every $f^{(n)} \in S_{\mathbb{C}}^{\widehat{\otimes} n}, n \in \mathbb{N}_{0}$, is an analytical vector of each $a(\xi)$, i.e., the series

$$
\sum_{m=0}^{\infty} \frac{\left\|a(\xi)^{m} f^{(n)}\right\|_{\mathcal{F}_{\text {Ext }}(\mathcal{H})}}{m !}|z|^{m}, \quad z \in \mathbb{C},
$$

has a positive radius of convergence.

To this end, we define, for each $p \geq 1$ and $k \in \mathbb{N}_{0}$, a Hilbert space

$$
\mathcal{F}_{1, k}\left(S_{p}\right)=\bigoplus_{n=0}^{\infty} S_{p, \mathbb{C}}^{\widehat{\otimes} n}(n !)^{2} 2^{n k},
$$

i.e., for $f=\left(f^{(0)}, f^{(1)}, f^{(2)}, \ldots\right) \in \mathcal{F}_{1, k}\left(S_{p}\right)$,

$$
\|f\|_{\mathcal{F}_{1, k}\left(S_{p}\right)}^{2}=\sum_{n=0}^{\infty}\left|f^{(n)}\right|_{p}^{2}(n !)^{2} 2^{n k} .
$$

Because of (10), we have that topologically $\mathcal{F}_{1, k_{0}}\left(S_{1}\right) \subset \mathcal{F}_{\text {Ext }}(\mathcal{H})$ if $2^{k_{0} / 2} \geq K_{1} C_{1}$ and

$$
\|\cdot\|_{\mathcal{F}_{1, k_{0}}\left(S_{1}\right)} \geq\|\cdot\|_{\mathcal{F}_{\text {Ext }}(\mathcal{H})} .
$$

Taking to notice that each $a(\xi)^{m} f^{(n)}$ belongs to $\mathcal{F}_{\text {fin }}(S)$, it suffices to prove that the series

$$
\sum_{m=0}^{\infty} \frac{\left\|a(\xi)^{m} f^{(n)}\right\|_{\mathcal{F}_{1, k_{0}}\left(S_{1}\right)}}{m !}|z|^{m}
$$

has a positive radius of convergence. We have

$$
\begin{aligned}
a(\xi)^{m} & =\left(a^{+}(\xi)+2 a^{0}(\xi)+\langle\xi\rangle \mathrm{id}+a_{1}^{-}(\xi)+a_{2}^{-}(\xi)\right)^{m} \\
& =\sum_{j=1}^{5^{m}} Y_{j, m}(\xi)
\end{aligned}
$$


where every operator $Y_{j, m}(\xi)$ is a composition of $m$ operators every one of which is one of the operators $a^{+}(\xi), 2 a^{0}(\xi),\langle\xi\rangle \mathrm{id}, a_{1}^{-}(\xi), a_{2}^{-}(\xi)$. From (14)-(16), we deduce that

$$
\begin{aligned}
\left\|a^{+}(\xi) f^{(n)}\right\|_{\mathcal{F}_{1, k_{0}}\left(S_{1}\right)} & \leq(n+1) 2^{k_{0} / 2}|\xi|_{1}\left\|f^{(n)}\right\|_{\mathcal{F}_{1, k_{0}}\left(S_{1}\right)} \\
\left\|2 a^{0}(\xi) f^{(n)}\right\|_{\mathcal{F}_{1, k_{0}}\left(S_{1}\right)} & \leq 2 n C_{1}|\xi|_{1}\left\|f^{(n)}\right\|_{\mathcal{F}_{1, k_{0}}\left(S_{1}\right)} \\
\left\|a_{1}^{-}(\xi) f^{(n)}\right\|_{\mathcal{F}_{1, k_{0}}\left(S_{1}\right)} & \leq 2^{-k_{0} / 2}|\xi|_{-1}\left\|f^{(n)}\right\|_{\mathcal{F}_{1, k_{0}}\left(S_{1}\right)} \\
\left\|a_{2}^{-}(\xi) f^{(n)}\right\|_{\mathcal{F}_{1, k_{0}}\left(S_{1}\right)} & \leq(n-1) C_{1}^{2} 2^{-k_{0} / 2}|\xi|_{1}\left\|f^{(n)}\right\|_{\mathcal{F}_{1, k_{0}}\left(S_{1}\right)}
\end{aligned}
$$

Hence,

$$
\left\|a(\xi)^{m} f^{(n)}\right\|_{\mathcal{F}_{1, k_{0}}\left(S_{1}\right)} \leq 5^{m}\left(2 C_{1}^{2} 2^{k_{0} / 2}\right)^{m} \frac{(n+m) !}{n !} \max \left\{|\xi|_{1},\langle\xi\rangle\right\}\left\|f^{(n)}\right\|_{\mathcal{F}_{1, k_{0}}\left(S_{1}\right)} .
$$

Thus, it remains to note that the series

$$
\sum_{m=0}^{\infty} \frac{\left(10 C_{1}^{2} 2^{k_{0} / 2}\right)^{m}(n+m) !}{m !}|z|^{m}
$$

converges as $|z|<\left(10 C_{1}^{2} 2^{k_{0} / 2}\right)^{-1}$. By using, e.g., [5], Ch. 5, Th. 1.7, we conclude that the operators $a(\xi)$ are essentially selfadjoint on $\mathcal{F}_{\text {fin }}(S)$.

Let us show that the operators $a(\xi)$ commute on $\mathcal{F}_{\text {fin }}(S)$. Any operators $a^{+}\left(\xi_{1}\right)$ and $a^{+}\left(\xi_{2}\right)$ evidently commute. Hence, their adjoints $a^{-}\left(\xi_{1}\right)$ and $a^{-}\left(\xi_{2}\right)$ also commute. Next, the operators of second quantization $a^{0}\left(\xi_{1}\right)$ and $a^{0}\left(\xi_{2}\right)$ commute, since do the operators of multiplication by $\xi_{1}$ and $\xi_{2}$. Next, we have to show that

$$
a^{+}\left(\xi_{1}\right) a^{0}\left(\xi_{2}\right)+a^{0}\left(\xi_{1}\right) a^{+}\left(\xi_{2}\right)=a^{+}\left(\xi_{2}\right) a^{0}\left(\xi_{1}\right)+a^{0}\left(\xi_{2}\right) a^{+}\left(\xi_{1}\right)
$$

which evidently yields

$$
a^{-}\left(\xi_{1}\right) a^{0}\left(\xi_{2}\right)+a^{0}\left(\xi_{1}\right) a^{-}\left(\xi_{2}\right)=a^{-}\left(\xi_{2}\right) a^{0}\left(\xi_{1}\right)+a^{0}\left(\xi_{2}\right) a^{-}\left(\xi_{1}\right) .
$$

But (19) can be easily verified on the vectors of the form $\varphi^{\otimes n}, \varphi \in S$, if one uses the equality

$$
\begin{aligned}
a^{0}(\xi) \varphi_{1} \widehat{\otimes} \varphi_{2} \widehat{\otimes} \cdots \widehat{\otimes} \varphi_{n}= & \left(\xi \varphi_{1}\right) \widehat{\otimes} \varphi_{2} \widehat{\otimes} \cdots \widehat{\otimes} \varphi_{n} \\
& +\varphi_{1} \widehat{\otimes}\left(\xi \varphi_{2}\right) \widehat{\otimes} \cdots \widehat{\otimes} \varphi_{n}+\cdots+\varphi_{1} \widehat{\otimes} \cdots \widehat{\otimes} \varphi_{n-1} \widehat{\otimes}\left(\xi \varphi_{n}\right),
\end{aligned}
$$

which gives, in particular,

$$
a^{0}(\xi) \varphi_{1} \widehat{\otimes} \varphi_{2}^{\otimes(n-1)}=\left(\xi \varphi_{1}\right) \widehat{\otimes} \varphi_{2}^{\otimes(n-1)}+(n-1) \varphi_{1} \widehat{\otimes}\left(\xi \varphi_{2}\right) \widehat{\otimes} \varphi_{2}^{\otimes(n-2)} .
$$

Finally, we have to show that

$$
a^{+}\left(\xi_{1}\right) a^{-}\left(\xi_{2}\right)+a^{-}\left(\xi_{1}\right) a^{+}\left(\xi_{2}\right)=a^{+}\left(\xi_{2}\right) a^{-}\left(\xi_{1}\right)+a^{-}\left(\xi_{2}\right) a^{+}\left(\xi_{1}\right) .
$$


It is not hard to see that

$$
a_{2}^{-}(\xi) \varphi_{1} \widehat{\otimes} \varphi_{2}^{\otimes n}=2 n\left(\xi \varphi_{1} \varphi_{2}\right) \widehat{\otimes} \varphi_{2}^{\otimes(n-1)}+n(n-1) \varphi_{1} \widehat{\otimes}\left(\xi \varphi_{2}^{2}\right) \widehat{\otimes} \varphi_{2}^{\otimes(n-2)} .
$$

Then,

$$
\begin{aligned}
& \left(a^{+}\left(\xi_{1}\right) a^{-}\left(\xi_{2}\right)+a^{-}\left(\xi_{1}\right) a^{+}\left(\xi_{2}\right)\right) \varphi^{\otimes n} \\
& =n\left\langle\xi_{2}, \varphi\right\rangle \xi_{1} \widehat{\otimes} \varphi^{\otimes(n-1)}+n(n-1) \xi_{1} \widehat{\otimes}\left(\xi_{2} \varphi^{2}\right) \widehat{\otimes} \varphi^{\otimes(n-2)}+\left\langle\xi_{1}, \xi_{2}\right\rangle \varphi^{\otimes n} \\
& \quad+n\left\langle\xi_{1}, \varphi\right\rangle \xi_{2} \widehat{\otimes} \varphi^{\otimes(n-1)}+2 n\left(\xi_{1} \xi_{2} \varphi\right) \widehat{\otimes} \varphi^{\otimes(n-1)}+n(n-1) \xi_{2} \widehat{\otimes}\left(\xi_{1} \varphi^{2}\right) \widehat{\otimes} \varphi^{\otimes(n-2)},
\end{aligned}
$$

which is symmetric in $\xi_{1}$ and $\xi_{2}$. Thus, arbitrary $a\left(\xi_{1}\right)$ and $a\left(\xi_{2}\right)$ commute.

Since the operators $a^{\sim}(\xi)$ are essentially selfadjoint on $\mathcal{F}_{\text {fin }}(S)$, the set

$$
\left(a^{\sim}(\xi)-z \mathrm{id}\right) \mathcal{F}_{\text {fin }}(S), \quad z \in \mathbb{C}, \Im z \neq 0,
$$

is dense in $\mathcal{F}_{\text {Ext }}(\mathcal{H})$. In addition

$$
\left(a^{\sim}(\xi)-z \mathrm{id}\right) \mathcal{F}_{\text {fin }}(S) \subset \mathcal{F}_{\text {fin }}(S) .
$$

Therefore, for arbitrary $\xi_{1}, \xi_{2} \in S$, the operators $a^{\sim}\left(\xi_{1}\right), a^{\sim}\left(\xi_{2}\right)$, and

$$
a^{\sim}\left(\xi_{1}\right) \uparrow\left(a^{\sim}\left(\xi_{2}\right)-z \mathrm{id}\right) \mathcal{F}_{\text {fin }}(S)
$$

have a total set of analytical vectors. Thus, by [5], Ch. 5, Th. 1.15, the operators commute in the sense of the resolutions of the identity.

Theorem 1 There exists a unitary isomorphism

$$
I: \mathcal{F}_{\mathrm{Ext}}(\mathcal{H}) \rightarrow L^{2}\left(S^{\prime}, \mathcal{B}\left(S^{\prime}\right), d \mu_{\mathrm{G}}\right)=\left(L_{\mathrm{G}}^{2}\right)
$$

between the extended Fock space $\mathcal{F}_{\text {Ext }}(\mathcal{H})$ and the complex $L^{2}$-space over $S^{\prime}$ with the Borel measure $\mu_{\mathrm{G}}$ of Gamma white noise, whose Laplace transform is given by (1). This isomorphism is defined on the dense set $\mathcal{F}_{\text {fin }}(S)$ by the formula

$$
\mathcal{F}_{\text {fin }}(S) \ni f=\left(f^{(n)}\right)_{n=0}^{\infty} \mapsto I f=(I f)(\omega)=\sum_{n=0}^{\infty}\left\langle: \omega^{\otimes n}{ }_{{ }_{\mathrm{G}}}, f^{(n)}\right\rangle
$$

(the series in (21) is actually finite), where $: \omega^{\otimes n}:_{\mathrm{G}} \in S^{\prime} \widehat{\otimes} n$ is the $n$-th Gamma-Wick power of $\omega \in S^{\prime}$ given by the recurrence formula

$$
\begin{gathered}
: \omega^{\otimes(n+1)}:_{\mathrm{G}}=: \omega^{\otimes(n+1)}:_{\mathrm{G}}\left(x_{1}, \ldots, x_{n+1}\right)=\left(: \omega^{\otimes n}:_{\mathrm{G}}\left(x_{1}, \ldots, x_{n}\right) \omega\left(x_{n+1}\right)\right)^{\sim} \\
-n\left(: \omega^{\otimes(n-1)}:_{\mathrm{G}}\left(x_{1}, \ldots, x_{n-1}\right) \delta\left(x_{n+1}-x_{n}\right)\right)^{\sim} \\
-n(n-1)\left(: \omega^{\otimes(n-1)}:_{\mathrm{G}}\left(x_{1}, \ldots, x_{n-1}\right) \delta\left(x_{n}-x_{n-1}\right) \delta\left(x_{n+1}-x_{n}\right)\right)^{\sim} \\
-2 n\left(: \omega^{\otimes n}:_{\mathrm{G}}\left(x_{1}, \ldots, x_{n}\right) \delta\left(x_{n+1}-x_{n}\right)\right)^{\sim}-\left(: \omega^{\otimes n}:_{\mathrm{G}}\left(x_{1}, \ldots, x_{n}\right) 1\left(x_{n+1}\right)\right)^{\sim}, \\
: \omega^{\otimes 0}:_{\mathrm{G}}=1,: \omega^{\otimes 1}:_{\mathrm{G}}=\omega-1,
\end{gathered}
$$


where $(\cdot)^{\sim}$ denotes the symmetrization of a function.

The image of each operator $a^{\sim}(\xi), \xi \in S$, under $I$ is the operator of multiplication by the monomial $\langle\cdot, \xi\rangle$ in $\left(L_{\mathrm{G}}^{2}\right)$ :

$$
I a^{\sim}(\xi) I^{-1}=\langle\cdot, \xi\rangle \cdot
$$

Remark 4. Let us explain the term "Gamma noise," introduced in [22]. Let $\Delta$ be a bounded Borel set in $\mathbb{R}^{d}$ and let $\chi_{\Delta}$ denote the indicator of $\Delta$. Then, one can define the random variable (r.v.) $X_{\Delta}=X_{\Delta}(\omega)=\left\langle\omega, \chi_{\Delta}\right\rangle$ as an $\left(L_{\mathrm{G}}^{2}\right)$-limit of a sequence of r.v.'s $\left\langle\omega, \xi_{j}\right\rangle$ such that $\xi_{j} \rightarrow \chi_{\Delta}$ in $L^{2}\left(\mathbb{R}^{d}, d \sigma\right)$ as $j \rightarrow \infty$. Then, the Laplace transform

$$
\int_{S^{\prime}} \exp \left[\lambda X_{\Delta}(\omega)\right] d \mu_{\mathrm{G}}(\omega)=\exp [-\sigma(\Delta) \log (1-\lambda)], \quad \lambda<1,
$$

coincides with the Laplace transform of a r.v. having Gamma distribution with parameter $\sigma(\Delta)$, i.e., the density of the distribution of $X_{\Delta}$ is

$$
p_{\Delta}(s)=\frac{s^{\sigma(\Delta)-1} e^{-s}}{\Gamma(\sigma(\Delta))}, \quad s>0 .
$$

In case $d=1$ and $d \sigma(x)=d x$, the process $\left(X_{t}\right)_{t \geq 0}$, where $X_{t}=X_{[0, t]}$ and $X_{0}=0$, is known as a Gamma process, see, e.g., [32], Sect. 19. Thus, the triple $\left(S^{\prime}, \mathcal{B}\left(S^{\prime}\right), \mu_{\mathrm{G}}\right)$ is a direct representation of the generalized stochastic process $\left(\dot{X}_{t}\right)_{t \geq 0}$, see [10]. So, the term "Gamma noise" is natural for $\mu_{\mathrm{G}}$.

Let us also note that the Gamma white noise is a special case of a compound Poisson white noise, see e.g. [9, 10, 26, 22], because the Laplace transform (1) can also be presented in the form

$$
\int_{S^{\prime}} \exp [\langle\omega, \varphi\rangle] d \mu_{\mathrm{G}}(\omega)=\exp \left[\int_{0}^{\infty} \int_{\mathbb{R}^{d}}\left(e^{s \varphi(x)}-1\right) d \sigma(x) \frac{e^{-s}}{s} d s\right] .
$$

This yields, in particular, that the measure $\mu_{\mathrm{G}}$ is concentrated on the following subset of $S^{\prime}$, e.g., $[15,17]$. Let $\Gamma$ denote the marked configuration space over supp $\sigma$ with marks in ]0, $\infty$ [ that is defined as follows: $\Gamma$ consists of sets $\gamma \subset \operatorname{supp} \sigma \times] 0, \infty[$ such that, for arbitrary disjoint $\left(x, s_{x}\right),\left(y, s_{y}\right) \in \gamma$, we have $x \neq y$, and for any $a>0$ and $K \subset \mathbb{R}^{d}$ compact, $\gamma \cap\left(K \times\left[a, \infty[)\right.\right.$ is a finite set. Then, the measure $\mu_{\mathrm{G}}$ is concentrated on the set of those $\omega \in S^{\prime}$ which can be presented in the form

$$
\omega=\sum_{\left(x, s_{x}\right) \in \gamma} s_{x} \delta_{x}
$$

with $\gamma \in \Gamma$. 
Proof of Theorem 1. Just as in the case of Gaussian and Poisson measures, see [3, 6, 24], we deduce, by using the projection spectral theorem [5], Ch. 3, Th. 2.7, the existence of a unitary isomorphism $I$ between $\mathcal{F}_{\text {Ext }}(\mathcal{H})$ and an $L^{2}$-space $L^{2}\left(S^{\prime}, \mathcal{B}\left(S^{\prime}\right), d \mu\right)$ for which the formulas (21)-(23) hold. Here, $\mu$ is a Borel probability measure on $S^{\prime}$, which is the spectral measure of the family $\left(a^{\sim}(\xi)\right)_{\xi \in S}$. Indeed, the major step in the proof of this fact is the following lemma.

Lemma 2 1) The family $\left(a^{\sim}(\xi)\right)_{\xi \in S}$ is connected with (9) in a standard way, i.e., for each $\xi \in S, a(\xi)$ is a linear continuous operator on $\mathcal{F}_{\text {fin }}(S)$.

2) For an arbitrary fixed $f \in \mathcal{F}_{\text {fin }}(S)$, the mapping

$$
S \ni \xi \mapsto a(\xi) f \in \mathcal{F}_{\text {fin }}(S)
$$

is linear and continuous.

3) The vacuum $\Omega=(1,0,0, \ldots) \in \mathcal{F}_{\text {fin }}(S)$ is a strong cyclic vector of the family $\left(a^{\sim}(\xi)\right)_{\xi \in S}$, i.e., the linear span of the set

$$
\{\Omega\} \cup\left\{a\left(\xi_{1}\right) \cdots a\left(\xi_{n}\right) \Omega \mid \xi_{i} \in S, i=1, \ldots, n, n \in \mathbb{N}\right\}
$$

is dense in $\mathcal{F}_{\text {fin }}(S)$.

The item 1 of Lemma 2 we have already proved, while the item 2 follows from the definition of the operators $a(\xi)$ and the estimates (14)-(16). The item 3 is implied by the fact that we have the standard creation operators, which allows us just to repeat the proof of the corresponding fact in the Gaussian and Poisson cases.

Thus, having Lemma 2, we apply the projection spectral theorem, which gives us the existence of a spectral measure $\mu$ of the family $\left(a^{\sim}(\xi)\right)_{\xi \in S}$ as a probability measure on $S^{\prime}$. For $\mu$-almost every $\omega \in S^{\prime}$, there exists a generalized joint eigenvector

$$
P(\omega)=\left(P^{(n)}(\omega)\right)_{n=0}^{\infty} \in \mathcal{F}_{\text {fin }}^{*}(S)
$$

satisfying

$$
\langle\langle P(\omega), a(\xi) f\rangle\rangle=\langle\omega, \xi\rangle\langle\langle P(\omega), f\rangle\rangle, \quad f \in \mathcal{F}_{\text {fin }}(S) .
$$

Then, the operator $I$ defined by

$$
\begin{gathered}
I f=(I f)(\omega)=\langle\langle P(\omega), f\rangle\rangle=\sum_{n=0}^{\infty}\left\langle P^{(n)}(\omega), f^{(n)}\right\rangle n !, \\
f=\left(f^{(n)}\right)_{n=0}^{\infty} \in \mathcal{F}_{\text {fin }}(S),
\end{gathered}
$$

can be extended by continuity to a unitary operator between $\mathcal{F}_{\text {Ext }}(\mathcal{H})$ and $L^{2}\left(S^{\prime}, \mathcal{B}\left(S^{\prime}\right), d \mu\right)$, under which any operator $a^{\sim}(\xi)$ goes over into the operator of multiplication by $\langle\omega, \xi\rangle$ (see $(27)$ and $(28)$ ). Denoting $: \omega^{\otimes n}:_{\mathrm{G}}=P^{(n)}(\omega) n$ !, we 
present the unitary $I$ in the form (21). The recurrence formula (22) can now be derived from the equality

$$
\begin{gathered}
\langle\omega, \xi\rangle\left\langle: \omega^{\otimes n}:_{\mathrm{G}}, \xi^{\otimes n}\right\rangle=\left\langle: \omega^{\otimes(n+1)}:_{\mathrm{G}}, \xi^{\otimes(n+1)}\right\rangle+\left\langle: \omega^{\otimes n}:_{\mathrm{G}}, 2 n\left(\xi^{2}\right) \widehat{\otimes} \xi^{\otimes(n-1)}+\langle\xi\rangle \xi^{\otimes n}\right\rangle \\
+\left\langle: \omega^{\otimes(n-1)}:_{\mathrm{G}}, n\left\langle\xi^{2}\right\rangle \xi^{\otimes(n-1)}+n(n-1)\left(\xi^{3}\right) \widehat{\otimes} \xi^{\otimes(n-2)}\right\rangle .
\end{gathered}
$$

As easily seen, : $\omega^{\otimes n}:$ is well defined as an element of $S^{\prime \widehat{\otimes} n}$ for each $\omega \in S^{\prime}$, not only for $\mu$-almost all $\omega \in S^{\prime}$.

Thus, we need only to prove that $\mu$ is, in fact, the measure of Gamma white noise.

Let $\mathcal{B}_{\mathrm{b}}\left(\mathbb{R}^{d}\right)$ denote the set of all bounded Borel sets in $\mathbb{R}^{d}$, let $\Delta \in \mathcal{B}_{\mathrm{b}}\left(\mathbb{R}^{d}\right)$, and let $\chi_{\Delta}$ denote the indicator of $\Delta$. Denote by $a^{\sim}\left(\chi_{\Delta}\right)$ the operator in $\mathcal{F}_{\text {Ext }}(\mathcal{H})$ whose image under the unitary $I$ is the operator of multiplication by the function $\left\langle\omega, \chi_{\Delta}\right\rangle=\left\langle: \omega^{\otimes 1}:_{\mathrm{G}}, \chi_{\Delta}\right\rangle+\sigma(\Delta)=\left(I \chi_{\Delta}\right)(\omega)+\sigma(\Delta)$.

Each of the vectors $\chi_{\Delta}^{\otimes n}, n \geq 0, \chi_{\Delta}^{\otimes 0}:=\Omega$, belongs to $\mathcal{F}_{\text {Ext }}(\mathcal{H})$, and let $\mathcal{K}_{\Delta}$ be the subspace of $\mathcal{F}_{\text {Ext }}(\mathcal{H})$ spanned by these vectors. A limiting procedure shows that

$$
a^{\sim}\left(\chi_{\Delta}\right) \chi_{\Delta}^{\otimes n}=\chi_{\Delta}^{\otimes(n+1)}+(2 n+\sigma(\Delta)) \chi_{\Delta}^{\otimes n}+n(n-1+\sigma(\Delta)) \chi_{\Delta}^{\otimes(n-1)} .
$$

Therefore, $\mathcal{K}_{\Delta}$ is an invariant subspace for the operator $a^{\sim}\left(\chi_{\Delta}\right)$. Let $a_{\Delta}$ stand for the restriction of $a^{\sim}\left(\chi_{\Delta}\right)$ to the linear span of the vectors $\chi_{\Delta}^{\otimes n}$. Then, $a_{\Delta}$ is a densely defined, Hermitian operator in $\mathcal{K}_{\Delta}$.

Let $c_{n, \Delta}=\left\|\chi_{\Delta}^{\otimes n}\right\|_{\mathcal{F}_{\mathrm{Ext}}(\mathcal{H})}$, then the vectors $\left(e_{\Delta}^{(n)}\right)_{n=0}^{\infty}$, with $e_{\Delta}^{(n)}=c_{n, \Delta}^{-1} \chi_{\Delta}^{\otimes n}$, form an orthonormal basis $(\mathrm{ONB})$ in $\mathcal{K}_{\Delta}$. (29) yields

$$
a_{\Delta} e_{\Delta}^{(n)}=\frac{c_{n+1, \Delta}}{c_{n, \Delta}} e_{\Delta}^{(n+1)}+(2 n+\sigma(\Delta)) e_{\Delta}^{(n)}+n(n-1+\sigma(\Delta)) \frac{c_{n-1, \Delta}}{c_{n, \Delta}} e_{\Delta}^{(n-1)} .
$$

Since $a_{\Delta}$ is Hermitian, the matrix of this operator in the ONB $\left(e_{\Delta}^{(n)}\right)_{n=0}^{\infty}$ must be symmetric, which together with the formula (30) implies that this matrix is a Jacobi one, see [2], i.e.,

$$
a_{\Delta} e_{\Delta}^{(n)}=\alpha_{n+1, \Delta} e_{\Delta}^{(n+1)}+\beta_{n, \Delta} e_{\Delta}^{(n)}+\alpha_{n, \Delta} e_{\Delta}^{(n-1)} .
$$

Comparing (30) and (31) gives the equality

$$
\frac{c_{n, \Delta}}{c_{n-1, \Delta}}=n(n-1+\sigma(\Delta)) \frac{c_{n-1, \Delta}}{c_{n, \Delta}}
$$

from where

$$
\frac{c_{n, \Delta}}{c_{n-1, \Delta}}=\sqrt{n(n-1+\sigma(\Delta))} .
$$

Substituting (32) into (30), we derive that the coefficients in (31) equal

$$
\alpha_{n, \Delta}=\sqrt{n(n-1+\sigma(\Delta))}, \quad \beta_{n, \Delta}=2 n+\sigma(\Delta) .
$$


By using e.g. [2], Ch. 7, Th. 1.3, we conclude that the Jacobi matrix defined by the coefficients (33) has a unique spectral measure. This means that there exists a unique probability measure $\mu_{\Delta}$ on $\mathbb{R}$ such that the system of polynomials $\left(P_{n, \Delta}\right)_{n=0}^{\infty}$ defined by the recurrence formula

$$
P_{n+1, \Delta}(s)=\frac{1}{\alpha_{n+1, \Delta}}\left(\left(s-\beta_{n, \Delta}\right) P_{n, \Delta}(s)-\alpha_{n, \Delta} P_{n-1, \Delta}(s)\right)
$$

forms an ONB in $L^{2}\left(\mathbb{R}, d \mu_{\Delta}\right)$, and under the unitary $I_{\Delta}: \mathcal{K}_{\Delta} \rightarrow L^{2}\left(\mathbb{R}, d \mu_{\Delta}\right)$ given by $I_{\Delta} e_{\Delta}^{(n)}=P_{n, \Delta}$ the operator $a_{\Delta}$ - the closure in $\mathcal{K}_{\Delta}$ of the essentially selfadjoint operator $a_{\Delta}$ - goes over into the operator of multiplication by $s$. Moreover, the form of the coefficients (33) gives (see, e.g., [7]) that the measure $\mu_{\Delta}$ is exactly the Gamma distribution having density (24).

On the other hand, since $I a^{\sim}\left(\chi_{\Delta}\right) I^{-1}$ is the operator of multiplication by $\left\langle\omega, \chi_{\Delta}\right\rangle$, (31) and (34) imply that

$$
\left(I e_{\Delta}^{(n)}\right)(\omega)=P_{n, \Delta}\left(\left\langle\omega, \chi_{\Delta}\right\rangle\right) .
$$

Therefore, $\mu_{\Delta}$ coincides with the distribution of the r.v. $\left\langle\omega, \chi_{\Delta}\right\rangle$.

Next, for arbitrary disjoint sets $\Delta_{1}, \Delta_{2} \in \mathcal{B}_{\mathrm{b}}\left(\mathbb{R}^{d}\right)$, the spaces $\mathcal{K}_{\Delta_{1}} \ominus \widehat{\Omega}$ and $\mathcal{K}_{\Delta_{2}} \ominus \widehat{\Omega}$ are orthogonal in $\mathcal{F}_{\text {Ext }}(\mathcal{H})$. Here, $\widehat{\Omega}$ denotes the vacuum space spanned by $\Omega$, and $\ominus$ denotes the orthogonal difference. Therefore, the r.v.'s $\left\langle\omega, \chi_{\Delta_{1}}\right\rangle$ and $\left\langle\omega, \chi_{\Delta_{2}}\right\rangle$ are independent.

Thus, $\mu$ is indeed the measure of Gamma white noise.

Remark 5. Note that the polynomial $P_{n, \Delta}(s)$ is up to a multiplier $(-1)^{n}$ the normalized Laguerre polynomial with parameter $\sigma(\Delta)-1$. Therefore,

$$
\left\langle: \omega^{\otimes n}:_{\mathrm{G}}, f^{(n)}\right\rangle=(-1)^{n}\left\langle L_{n}^{\sigma}(\omega), f^{(n)}\right\rangle,
$$

where $L_{n}^{\sigma}(\omega)$ is the kernel of the generalized Laguerre polynomial introduced in [22]. This yields, in particular, that the Gamma-Wick exponential defined by

$$
: e^{\langle\omega, \varphi\rangle}:_{\mathrm{G}}=\sum_{n=0}^{\infty} \frac{1}{n !}\left\langle: \omega^{\otimes n}:{ }_{\mathrm{G}}, \varphi^{\otimes n}\right\rangle, \quad \varphi \in S_{\mathbb{C}},
$$

has the following representation, for $\varphi \in S$ such that $\varphi>-1$,

$$
: e^{\langle\omega, \varphi\rangle}:_{\mathrm{G}}=\exp \left[\left\langle\omega, \frac{\varphi}{\varphi+1}\right\rangle-\langle\log (1+\varphi)\rangle\right] .
$$

Remark 6. Following the tradition of quantum probability, e.g., [30], the family of the operators $\left(a^{\sim}\left(\chi_{\Delta}\right)\right)_{\Delta \in \Lambda}$ can be called a quantum Gamma process in the extended Fock space $\mathcal{F}_{\text {Ext }}(\mathcal{H})$. 


\section{Chaos decomposition of the Gamma space}

In this section, we will discuss some properties of the unitary $I$ and the space of Gamma white noise $\left(L_{\mathrm{G}}^{2}\right)$.

Denote by $\mathcal{P}\left(S^{\prime}\right)$ the set of continuous polynomials on $S^{\prime}$, i.e., functions on $S^{\prime}$ of the form

$$
\phi(\omega)=\sum_{i=0}^{n}\left\langle\omega^{\otimes i}, f^{(i)}\right\rangle, \quad f^{(i)} \in S_{\mathbb{C}}^{\widehat{\otimes} i}, \omega^{\otimes 0}=1, i \in \mathbb{N}_{0} .
$$

The greatest number $n$ for which $f^{(n)} \neq 0$ is called the power of a polynomial. Denote also by $\mathcal{P}_{n}\left(S^{\prime}\right)$ the set of continuous polynomials of power $\leq n$.

By using the isomorphism

$$
\mathcal{F}_{\text {fin }}(S) \ni f=\left(f^{(n)}\right)_{n=0}^{\infty} \mapsto \sum_{n=0}^{\infty}\left\langle\omega^{\otimes n}, f^{(n)}\right\rangle \in \mathcal{P}\left(S^{\prime}\right),
$$

one induces from $\mathcal{F}_{\text {fin }}(S)$ a topology on $\mathcal{P}\left(S^{\prime}\right)$, which makes it a nuclear space.

Proposition 1 We have

$$
I\left(\mathcal{F}_{\text {fin }}(S)\right)=\mathcal{P}\left(S^{\prime}\right),
$$

and the topology on $\mathcal{P}\left(S^{\prime}\right)$ induced from $\mathcal{F}_{\text {fin }}(S)$ by I coincides with that induced by (37).

Proof. Using the recurrence relation (22), one obtains by induction the inclusion $I\left(\mathcal{F}_{\text {fin }}(S)\right) \subset \mathcal{P}\left(S^{\prime}\right)$, and moreover, the equality

$$
\left\langle: \omega^{\otimes n}: \mathrm{G}, f^{(n)}\right\rangle=\left\langle\omega^{\otimes n}, f^{(n)}\right\rangle+p_{n-1}(\omega)
$$

where $p_{n-1}(\omega) \in \mathcal{P}_{n-1}\left(S^{\prime}\right)$. Using (38), one obtains again by induction the inverse inclusion $\mathcal{P}\left(S^{\prime}\right) \subset \mathcal{F}_{\text {fin }}(S)$. The statement about the topology on $\mathcal{P}\left(S^{\prime}\right)$ follows directly from the above consideration.

For every $n \in \mathbb{N}_{0}$, put $\left(L_{\mathrm{G}, n}^{2}\right)=I\left(\mathcal{F}_{\mathrm{Ext}}^{(n)}(\mathcal{H})\right)$, so that we obtain the following decomposition of the space $\left(L_{\mathrm{G}}^{2}\right)$ :

$$
\left(L_{\mathrm{G}}^{2}\right)=\bigoplus_{n=0}^{\infty}\left(L_{\mathrm{G}, n}^{2}\right)
$$

Proposition 2 For each $n \in \mathbb{N}$, we have

$$
\left(L_{\mathrm{G}, n}^{2}\right)=\mathcal{P}_{\mathrm{G}, n}\left(S^{\prime}\right) \ominus \mathcal{P}_{\mathrm{G}, n-1}\left(S^{\prime}\right),
$$

where $\mathcal{P}_{\mathrm{G}, n}\left(S^{\prime}\right)$ denotes the closure of $\mathcal{P}_{n}\left(S^{\prime}\right)$ in the $\left(L_{\mathrm{G}}^{2}\right)$ norm, and $\ominus$ stands for the orthogonal difference in $\left(L_{\mathrm{G}}^{2}\right)$. 
Proof. As appears from the proof of Proposition 1,

$$
I\left(\bigoplus_{i=0}^{n} S_{\mathbb{C}}^{\widehat{\otimes} i}\right)=\mathcal{P}_{n}\left(S^{\prime}\right)
$$

and hence

$$
I\left(\bigoplus_{i=0}^{n} \mathcal{F}_{\mathrm{Ext}}^{(n)}(\mathcal{H})\right)=\bigoplus_{i=0}^{n}\left(L_{\mathrm{G}, n}^{2}\right)=\mathcal{P}_{\mathrm{G}, n}\left(S^{\prime}\right),
$$

from where (40) follows.

Proposition 3 Let $P_{\mathrm{G}, n}$ stand for the orthogonal projection of $\left(L_{\mathrm{G}}^{2}\right)$ onto the sub$\operatorname{space}\left(L_{\mathrm{G}, n}^{2}\right)$. Then

$$
P_{\mathrm{G}, n}\left(\left\langle\omega^{\otimes n}, f^{(n)}\right\rangle\right)=\left\langle: \omega^{\otimes n}:_{\mathrm{G}}, f^{(n)}\right\rangle, \quad f^{(n)} \in S_{\mathbb{C}}^{\widehat{\otimes} n}
$$

Proof. (41) is derived from (38) and Proposition 2 by applying to the left and right hand sides of the latter formula the operator $P_{\mathrm{G}, n}$ and taking to notice that $\left\langle: \omega^{\otimes n}: \mathrm{G}, f^{(n)}\right\rangle \in\left(L_{\mathrm{G}, n}^{2}\right)$.

Propositions 2 and 3 make it possible to interpret (39) as a kind of chaos decomposition of $\left(L_{\mathrm{G}}^{2}\right)$. However, one should be careful with this term. Indeed, since $\mathbb{E}\left[X_{\Delta}\right]=\sigma(\Delta)$ and $\mathbb{E}\left[\left(X_{\Delta}-\sigma(\Delta)\right)^{2}\right]=\sigma(\Delta)$, the compensated Gamma process

$$
\tilde{X}_{\Delta}=\tilde{X}_{\Delta}(\omega)=X_{\Delta}(\omega)-\sigma(\Delta)=\left\langle\omega, \chi_{\Delta}\right\rangle-\sigma(\Delta)=\left\langle: \omega^{\otimes 1}:_{\mathrm{G}}, \chi_{\Delta}\right\rangle, \quad \Delta \in \mathcal{B}_{\mathrm{b}}\left(\mathbb{R}^{d}\right),
$$

is a normal martingale, and so one can introduce multiple stochastic integrals w.r.t. $\tilde{X}_{\Delta}$. But it follows from the general result of [8] that this process does not possess the chaotic representation property, so that (39) cannot be a chaos decomposition of $\left(L_{\mathrm{G}}^{2}\right)$ in the sense of multiple stochastic integrals. To be more precise, we present the following propopsition.

Proposition 4 The chaos in $\left(L_{\mathrm{G}}^{2}\right)$ generated by multiple stochastic integrals w.r.t. the compensated Gamma process $\tilde{X}_{\Delta}$ coincides with the image under the unitary $I$ of the usual Fock space $\mathcal{F}(\mathcal{H})$ as a subspace of $\mathcal{F}_{\text {Ext }}(\mathcal{H})$, and moreover

$$
\int_{\mathbb{R}^{d n}} f^{(n)}\left(x_{1}, \ldots, x_{n}\right) d \tilde{X}_{x_{1}} \cdots d \tilde{X}_{x_{n}}=I f^{(n)}
$$

for an arbitrary $f^{(n)} \in \mathcal{H}_{\mathbb{C}}^{\widehat{\otimes} n}$, where $\mathcal{H}_{\mathbb{C}}^{\widehat{\otimes} n}$ is considered as a subspace of $\mathcal{F}_{\text {Ext }}^{(n)}(\mathcal{H})$.

Proof. We remind that the $n$-fold stochastic integral w.r.t. $\tilde{X}_{\Delta}$ are defined as follows (e.g., $[30,8])$. First, one takes arbitrary disjoint sets $\Delta_{1}, \ldots, \Delta_{n} \in \Lambda$ and sets

$$
\int_{\mathbb{R}^{d n}}\left(\chi_{\Delta_{1}}\left(x_{1}\right) \cdots \chi_{\Delta_{n}}\left(x_{n}\right)\right)^{\sim} d \tilde{X}_{x_{1}} \cdots d \tilde{X}_{x_{n}}=\tilde{X}_{\Delta_{1}} \cdots \tilde{X}_{\Delta_{n}} .
$$


Then, one extends the equality (42) by linearity and continuity in the $\mathcal{H}_{\mathbb{C}}^{\widehat{\otimes} n}$ norm. But Theorem 1 yields that

$$
\begin{gathered}
\tilde{X}_{\Delta_{1}}(\omega) \cdots \tilde{X}_{\Delta_{n}}(\omega)=\left\langle: \omega^{\otimes 1}:_{\mathrm{G}}, \chi_{\Delta_{1}}\right\rangle \cdots\left\langle: \omega^{\otimes 1}:_{\mathrm{G}}, \chi_{\Delta_{n}}\right\rangle \\
=I\left(\left(a\left(\chi_{\Delta_{1}}\right)-\sigma\left(\Delta_{1}\right) \mathrm{id}\right) \cdots\left(a\left(\chi_{\Delta_{n}}\right)-\sigma\left(\Delta_{n}\right) \mathrm{id}\right) \Omega\right) \\
=I\left(a^{+}\left(\chi_{\Delta_{1}}\right) \cdots a^{+}\left(\chi_{\Delta_{n}}\right) \Omega\right)=I\left(\chi_{\Delta_{1}} \widehat{\otimes} \cdots \widehat{\otimes} \chi_{\Delta_{n}}\right),
\end{gathered}
$$

which together with our consideration at the end of Section 1 gives the statement.

\section{Spaces of test and generalized functions and coordinate operators on them}

In this section, we will construct spaces of test and generalized functions of Gamma white noise and consider some their properties. The simplest ones can be obtained by applying $I$ to (9). Thus, taking to notice Proposition 1, we get

$$
\left(L_{\mathrm{G}}^{2}\right) \supset \mathcal{P}\left(S^{\prime}\right), \quad \mathcal{P}\left(S^{\prime}\right)^{*} \supset \mathcal{P}\left(S^{\prime}\right) .
$$

The dual $\mathcal{P}\left(S^{\prime}\right)^{*}$ of the space of continuous polynomials $\mathcal{P}\left(S^{\prime}\right)$ consists of generalized functions, which we present in the form

$$
\Phi=\Phi(\omega)=\sum_{n=0}^{\infty}\left\langle: \omega^{\otimes n}:{ }_{\mathrm{G}}, F^{(n)}\right\rangle,
$$

where $F=\left(F^{(n)}\right)_{n=0}^{\infty} \in \mathcal{F}_{\text {fin }}^{*}(S)$ and the dualization of $\Phi$ with $\phi \in \mathcal{P}\left(S^{\prime}\right), \phi(\omega)=$ $\sum_{n=0}^{\infty}\left\langle: \omega^{\otimes n}: \mathrm{G}, f^{(n)}\right\rangle$, is given by

$$
\langle\langle\Phi, \phi\rangle\rangle=\sum_{n=0}^{\infty}\left\langle\overline{F^{(n)}}, f^{(n)}\right\rangle n ! .
$$

However, the test space $\mathcal{P}\left(S^{\prime}\right)$ is too small. This is why we will consider the following nuclear space and its dual: (cf. [19, 1, 23, 20])

$$
\begin{gathered}
\mathcal{F}_{\text {Ext }}(\mathcal{H}) \supset \underset{p, k \rightarrow \infty}{\operatorname{proj} \lim } \mathcal{F}_{1, k}\left(S_{p}\right)=\mathcal{F}_{1}\left(S_{1}\right), \\
\mathcal{F}_{-1}\left(S^{\prime}\right)=\operatorname{ind}_{p, k \rightarrow \infty} \mathcal{F}_{-1,-k}\left(S_{-p}\right) \supset \underset{p, k \rightarrow \infty}{\operatorname{proj} \lim } \mathcal{F}_{1, k}\left(S_{p}\right)=\mathcal{F}_{1}\left(S_{1}\right),
\end{gathered}
$$

where the spaces $\mathcal{F}_{1, k}\left(S_{p}\right), p \geq 1, k \geq k_{0}$, are defined by $(18), \mathcal{F}_{-1,-k}\left(S_{-p}\right)$ are their respective duals (w.r.t. $\mathcal{F}(\mathcal{H})$ ):

$$
\mathcal{F}_{-1,-k}\left(S_{-p}\right)=\bigoplus_{n=0}^{\infty} S_{-p, \mathbb{C}}^{\widehat{\otimes} n} 2^{-n k}
$$


The application of $I$ to (43) gives the following spaces of test and generalized functions:

$$
\begin{aligned}
& \left(L_{\mathrm{G}}^{2}\right) \supset \underset{p, k \rightarrow \infty}{\operatorname{proj} \lim }\left(S_{\mathrm{G}}\right)_{p, k}^{1}=\left(S_{\mathrm{G}}\right)^{1}, \\
& \left(S_{\mathrm{G}}\right)^{-1}=\operatorname{ind}_{p, k \rightarrow \infty} \lim _{\mathrm{G}}\left(S_{\mathrm{G}}\right)_{-p,-k}^{-1} \supset \underset{p, k \rightarrow \infty}{\operatorname{proj} \lim }\left(S_{\mathrm{G}}\right)_{p, k}^{1}=\left(S_{\mathrm{G}}\right)^{1} .
\end{aligned}
$$

On the space $\left(S_{\mathrm{G}}\right)^{-1}$, we introduce an $\mathcal{S}$-transform in a standard way (cf. [19, 1 , $23,20])$ : If $\Phi \in\left(S_{\mathrm{G}}\right)^{-1}$, then by the definition of $\left(S_{\mathrm{G}}\right)^{-1}$ there are $p \geq 1$ and $k \geq k_{0}$ such that $\Phi \in\left(S_{\mathrm{G}}\right)_{-p,-k}^{-1}$, and we put

$$
\mathcal{S}[\Phi](\theta)=\left\langle\left\langle\bar{\Phi},: e^{\langle\cdot, \theta\rangle}:_{\mathrm{G}}\right\rangle\right\rangle, \quad \theta \in S_{\mathbb{C}},|\theta|_{p}<2^{-k / 2},
$$

where $\bar{\Phi}$ is the complex conjugate of $\Phi$ and $: e^{\langle\cdot, \theta\rangle}:_{\mathrm{G}}$ is defined by (35). The condition on the norm of $\xi$ in (44) implies that $: e^{\langle\cdot, \theta\rangle}:_{\mathrm{G}} \in\left(S_{\mathrm{G}}\right)_{p, k}^{1}$, so that the dualization in $(44)$ is well-defined. From the definition of the $\mathcal{S}$-transform, we have for each

$$
\Phi=\Phi(\omega)=\sum_{n=0}^{\infty}\left\langle: \omega^{\otimes n}: \mathrm{G}, F^{(n)}\right\rangle
$$

that

$$
\mathcal{S}[\Phi](\theta)=\sum_{n=0}^{\infty}\left\langle F^{(n)}, \theta^{\otimes n}\right\rangle .
$$

Notice that if $\theta \in S_{\mathbb{C}}^{\prime}$, we can still define the Wick exponential $: e^{\langle\cdot, \theta\rangle}:_{\mathrm{G}}$ by the same formula (35), considering it as a generalized function from $\left(S_{\mathrm{G}}\right)^{-1}$. Hence, the $\mathcal{S}$-transform of a test function $\phi(\omega)=\sum_{n=0}^{\infty}\left\langle: \omega^{\otimes n}{ }_{\mathrm{G}}, f^{(n)}\right\rangle \in\left(S_{\mathrm{G}}\right)^{1}$ can be extended on $S_{\mathbb{C}}^{\prime}$ by setting

$$
\mathcal{S}[\phi](\theta)=\left\langle\left\langle\bar{\phi},: e^{\langle\cdot, \theta\rangle}:_{\mathrm{G}}\right\rangle\right\rangle=\sum_{n=0}^{\infty}\left\langle f^{(n)}, \theta^{\otimes n}\right\rangle, \quad \theta \in S_{\mathbb{C}}^{\prime} .
$$

The following two theorems, which are due to [19], give the description of the spaces $\left(S_{\mathrm{G}}\right)^{1}$ and $\left(S_{\mathrm{G}}\right)^{-1}$ in terms of the $\mathcal{S}$-transform.

Theorem 2 For any $\Phi \in\left(S_{\mathrm{G}}\right)^{-1}, \mathcal{S}[\Phi]$ is a function holomorphic at zero, i.e., $\mathcal{S}[\Phi] \in \operatorname{Hol}_{0}\left(S_{\mathbb{C}}\right)$. And conversely, for any $F \in \operatorname{Hol}_{0}\left(S_{\mathbb{C}}\right)$, there exists a unique $\Phi \in\left(S_{\mathrm{G}}\right)^{-1}$ such that $\mathcal{S}[\Phi]=F$ (i.e., there is a neighborhood of zero, $U$, such that $\mathcal{S}[\Phi](\theta)=F(\theta)$ for all $\theta \in U)$.

Denote by $\mathcal{E}_{\min }^{1}\left(S_{\mathbb{C}}^{\prime}\right)$ the set of entire functions on $S_{\mathbb{C}}^{\prime}$ of the first order of growth and of minimal type, i.e., a function $u$ entire on $S_{\mathbb{C}}^{\prime}$ belongs to $\mathcal{E}_{\min }^{1}\left(S_{\mathbb{C}}^{\prime}\right)$ iff

$$
\forall p>0 \forall \epsilon>0 \exists C>0: \quad|u(\theta)| \leq C e^{\epsilon|\theta|_{-p}}, \quad \theta \in S_{-p, \mathbb{C}}
$$


The set $\mathcal{E}_{\text {min }}^{1}\left(S_{\mathbb{C}}^{\prime}\right)$ can be topologized by the following family of norms

$$
|u|_{1, p, k}=\sup _{\theta \in S_{-p, \mathbb{C}}}\left\{|u(\theta)| \exp \left[-\frac{1}{k}|\theta|_{-p}\right]\right\}, \quad p, k \in \mathbb{N},
$$

so that $\mathcal{E}_{\min }^{1}\left(S_{\mathbb{C}}^{\prime}\right)$ becomes a countably normed space.

Theorem 3 For any $\phi \in\left(S_{\mathrm{G}}\right)^{1}, \mathcal{S}[\phi] \in \mathcal{E}_{\min }^{1}\left(S_{\mathbb{C}}^{\prime}\right)$. And conversely, for any $f \in$ $\mathcal{E}_{\text {min }}^{1}\left(S_{\mathbb{C}}^{\prime}\right)$, there exits a unique $\phi \in\left(S_{\mathrm{G}}\right)^{1}$ such that $\mathcal{S}[\phi]=f$. Moreover, the $\mathcal{S}$ transform is a homeomorphism between the topological spaces $\left(S_{\mathrm{G}}\right)^{1}$ and $\mathcal{E}_{\min }^{1}\left(\mathcal{S}_{\mathbb{C}}^{\prime}\right)$.

Denote now by $\mathcal{E}_{\text {min }}^{1}\left(S^{\prime}\right)$ the set of the restrictions to $S^{\prime}$ of functions from $\mathcal{E}_{\text {min }}^{1}\left(S_{\mathbb{C}}^{\prime}\right)$. The topology on $\mathcal{E}_{\min }^{1}\left(S^{\prime}\right)$ is induced by that of $\mathcal{E}_{\min }^{1}\left(S_{\mathbb{C}}^{\prime}\right)$.

The next theorem gives the inner description of the test space $\left(S_{\mathrm{G}}\right)^{1}$.

Theorem 4 We have

$$
\left(S_{\mathrm{G}}\right)^{1}=\mathcal{E}_{\min }^{1}\left(S^{\prime}\right)
$$

where (46) is understood as an equality of topological spaces.

Proof. This theorem is, in fact, a direct corollary of results of [20] (see also [27]), since the Gamma-Wick monomials $\left\langle: \omega^{\otimes n}:_{\mathrm{G}}, f^{(n)}\right\rangle$ form a generalized Appell system in the sense of [20] with the transformation function $\alpha(\varphi)=\frac{\varphi}{\varphi+1}$ (cf. [22]). However, for the further exposition, we need to state some details of the theory of (generalized) Appell polynomials.

Let $\mu$ be a probability measure on $\left(S^{\prime}, \mathcal{B}\left(S^{\prime}\right)\right)$ satisfying the following two conditions:

Condition 1. The Laplace transform $\ell_{\mu}(\varphi)$ of the measure $\mu$ can be extended to an analytic function in a neighborhood of zero in $S_{\mathbb{C}}^{\prime}$,

Condition 2. If $\phi \in \mathcal{P}\left(S^{\prime}\right)$ and $\phi=0 \mu$-almost everywhere, then $\phi \equiv 0$.

Put

$$
e_{\mu}(\varphi ; \omega)=\frac{e^{\langle\omega, \varphi\rangle}}{\ell_{\mu}(\varphi)}
$$

Due to Condition 1 and the fact that $\ell_{\mu}(0)=1$, for every fixed $\omega \in S^{\prime} e_{\mu}(\varphi ; \omega)$ is an analytic function of $\varphi$ in a neighborhood of zero.

Define now $P_{\mu}^{(0)}(\omega)=1$ and $P_{\mu}^{(n)}(\omega) \in S^{\prime \widehat{\otimes} n}, n \in \mathbb{N}$, by

$$
\left\langle P_{\mu}^{(n)}(\omega), \varphi^{\otimes n}\right\rangle=\left.\frac{d^{n}}{d t^{n}}\right|_{t=0} e_{\mu}(t \varphi ; \omega),
$$

so that

$$
e_{\mu}(\varphi ; \omega)=\sum_{n=0}^{\infty} \frac{1}{n !}\left\langle P_{\mu}^{(n)}(\omega), \varphi^{\otimes n}\right\rangle
$$


By using the kernel theorem, one shows that this definition is correct, i.e., that (47) really determines an element of $S^{\prime \widehat{\otimes} n}$. A function of $\omega$ of the form $\left\langle P_{\mu}^{(n)}(\omega), f^{(n)}\right\rangle$, where $f^{(n)} \in S_{\mathbb{C}}^{\widehat{\otimes} n}$, is called an Appell polynomial. These polynomials have the following very important property: let $v \in S^{\prime}$ and let $D_{v}$ denote the Gâteaux derivative in direction $v$ :

$$
D_{v} \phi(\omega)=\left.\frac{d}{d t}\right|_{t=0} \phi(\omega+t v)=\lim _{t \rightarrow 0} \frac{\phi(\omega+t v)-\phi(\omega)}{t},
$$

then

$$
D_{v}\left\langle P_{\mu}^{(n)}(\omega), \varphi^{\otimes n}\right\rangle=n\langle v, \varphi\rangle\left\langle P_{\mu}^{(n-1)}(\omega), \varphi^{\otimes(n-1)}\right\rangle,
$$

i.e., $D_{v}$ acts as an annihilation operator w.r.t. the Appell polynomials. Particularly, for the gradient

$$
\nabla_{x}=D_{\delta_{x}}, \quad x \in \mathbb{R}^{d}
$$

we have

$$
\nabla_{x}\left\langle P_{\mu}^{(n)}(\omega), \varphi^{\otimes n}\right\rangle=n \varphi(x)\left\langle P_{\mu}^{(n-1)}(\omega), \varphi^{\otimes(n-1)}\right\rangle
$$

and so

$$
\nabla_{x} e_{\mu}(\varphi ; \omega)=\varphi(x) e_{\mu}(\varphi ; \omega) .
$$

Now, for each $p \geq 1$ and $k \in \mathbb{N}_{0}$, one introduces a norm $\|\cdot\|_{1, p, k}$ on $\mathcal{P}\left(S^{\prime}\right)$ as follows: for

$$
\phi(\omega)=\sum_{n=0}^{k}\left\langle P_{\mu}^{(n)}(\omega), f^{(n)}\right\rangle
$$

we have

$$
\|\phi\|_{1, p, k}^{2}=\sum_{n=0}^{\infty}\left|f^{(n)}\right|_{p}^{2}(n !)^{2} 2^{n k},
$$

and let $\left[S_{\mu}\right]_{p, k}^{1}$ denote the closure of $\mathcal{P}\left(S^{\prime}\right)$ in this norm. Put

$$
\left[S_{\mu}\right]^{1}=\underset{p, k \rightarrow \infty}{\operatorname{proj}} \lim \left[S_{\mu}\right]_{p, k}^{1} .
$$

Thus, one has [23] that there exist $p_{1} \geq 1$ and $k_{1} \in \mathbb{N}_{0}$ such that $\left[S_{\mu}\right]_{p_{1}, k_{1}}^{1}$ is topologically embedded into $L^{2}\left(S^{\prime}, d \mu\right)=\left(L_{\mu}^{2}\right)$, and moreover, one has the topological equality

$$
\left[S_{\mu}\right]^{1}=\mathcal{E}_{\min }^{1}\left(S^{\prime}\right)
$$

Next, we proceed to consider a generalized Appell system. Let $\alpha$ be an arbitrary function that maps a neighborhood of zero in $S_{\mathbb{C}}$ into itself, which is supposed to be holomorphic, invertible and to satisfy $\alpha(0)=0$.

Let

$$
e_{\mu, \alpha}(\varphi ; \omega)=e_{\mu}(\alpha(\varphi) ; \omega)=\frac{\left.e^{\langle\omega, \alpha(\varphi)}\right\rangle}{\ell_{\mu}(\alpha(\varphi))}
$$


Then, analogously to the above, one defines generalized Appell polynomials $\left\langle P_{\mu, \alpha}^{(n)}(\omega), f^{(n)}\right\rangle$ in such a way that

$$
e_{\mu, \alpha}(\varphi ; \omega)=\sum_{n=0}^{\infty} \frac{1}{n !}\left\langle P_{\mu, \alpha}^{(n)}(\omega), \varphi^{\otimes n}\right\rangle .
$$

The $\alpha$-gradient $\nabla_{x}^{\alpha}$ is defined now by

$$
\nabla_{x}^{\alpha}\left\langle P_{\mu, \alpha}^{(n)}(\omega), \varphi^{\otimes n}\right\rangle=n \varphi(x)\left\langle P_{\mu, \alpha}^{(n-1)}(\omega), \varphi^{\otimes(n-1)}\right\rangle,
$$

so that

$$
\nabla_{x}^{\alpha} e_{\mu, \alpha}(\varphi ; \omega)=\varphi(x) e_{\mu, \alpha}(\varphi ; \omega) .
$$

Using the polynomials $\left\langle P_{\mu, \alpha}^{(n)}(\omega), f^{(n)}\right\rangle$, one defines just as above the spaces $\left[S_{\mu}\right]_{p, k, \alpha}^{1}$, and again [20] there exist $p_{2} \geq 1$ and $k_{2} \in \mathbb{N}_{0}$ such that $\left[S_{\mu}\right]_{p_{2}, k_{2}, \alpha}^{1}$ is topologically embedded into $\left(L_{\mu}^{2}\right)$ and

$$
\left[S_{\mu}\right]_{\alpha}^{1}=\underset{p, k \rightarrow \infty}{\operatorname{proj} \lim }\left[S_{\mu}\right]_{p, k, \alpha}^{1}=\mathcal{E}_{\min }^{1}\left(S^{\prime}\right) .
$$

Let us come back to the case of the Gamma measure $\mu=\mu_{\mathrm{G}}$. Evidently the Laplace transform $\ell_{\mathrm{G}}(\varphi)$ of $\mu_{\mathrm{G}}$ satisfies Condition 1 (see (1)). Proposition 1 implies that Condition 2 is also satisfied for $\mu_{\mathrm{G}}$. Thus, we can put

$$
e_{\mathrm{G}}(\varphi ; \omega)=\frac{\left.e^{\langle\omega, \varphi}\right\rangle}{\ell_{\mathrm{G}}(\varphi)}=\exp [\langle\omega, \varphi\rangle+\langle\log (1-\varphi)\rangle] .
$$

By setting

$$
\alpha(\varphi)=\frac{\varphi}{\varphi+1},
$$

which satisfies the conditions on $\alpha$, we get

$$
e_{\mathrm{G}, \alpha}(\varphi ; \omega)=\exp \left[\left\langle\omega, \frac{\varphi}{\varphi+1}\right\rangle-\langle\log (1+\varphi)\rangle\right] .
$$

Comparing the last formula with (36), we conclude that

$$
\left\langle: \omega^{\otimes n}:_{\mathrm{G}}, f^{(n)}\right\rangle=\left\langle P_{\mathrm{G}, \alpha}^{(n)}(\omega), f^{(n)}\right\rangle,
$$

which yields the theorem.

On the space $\left(S_{\mathrm{G}}\right)^{-1}$, one can introduce a Wick product $\diamond$ as follows (cf. [19, 23, $20])$ : for arbitrary $\Phi, \Psi \in\left(S_{\mathrm{G}}\right)^{-1}, \Phi \diamond \Psi$ is an element of $\left(S_{\mathrm{G}}\right)^{-1}$ such that

$$
\mathcal{S}[\Phi \diamond \Psi](\theta)=\mathcal{S}[\Phi](\theta) \mathcal{S}[\Psi](\theta)
$$


for $\theta \in S_{\mathbb{C}}$ from some neighborhood of zero. Since $\operatorname{Hol}_{0}\left(S_{\mathbb{C}}\right)$ is an algebra under pointwise multiplication of functions, this definition is correct. (52) implies that

$$
\left\langle: \omega^{\otimes n}:_{\mathrm{G}}, F^{(n)}\right\rangle \diamond\left\langle: \omega^{\otimes m}:_{\mathrm{G}}, G^{(m)}\right\rangle=\left\langle: \omega^{\otimes(n+m)}:_{\mathrm{G}}, F^{(n)} \widehat{\otimes} G^{(m)}\right\rangle .
$$

Notice that the Wick product of two test functions from $\left(S_{\mathrm{G}}\right)^{1}$ belongs again to $\left(S_{\mathrm{G}}\right)^{1}$.

Remark 7 . Because of (49)-(51), the above introduced Wick product on $\left(S_{\mathrm{G}}\right)^{-1}$ coincides with the Wick product on this space defined in the framework of biorthogonal analysis [23], i.e., by using the function $e_{\mathrm{G}}(\cdot ; \theta)$ instead of $: e^{\langle\cdot, \theta\rangle}:_{\mathrm{G}}$.

Let us consider a pair of simplest examples of generalized functions from $\left(S_{\mathrm{G}}\right)^{-1}$. The first one is the Gamma white noise:

$$
\omega(x)=: \omega::_{\mathrm{G}}(x)+1=\left\langle: \omega^{\otimes 1}:_{\mathrm{G}}, \delta_{x}\right\rangle+1 \in\left(S_{\mathrm{G}}\right)_{-1,-k_{0}}^{-1}, \quad x \in \operatorname{supp} \sigma,
$$

the function $\omega(x)-1=: \omega:_{\mathrm{G}}(x)$ can be thought of as the compensated Gamma white noise. Next, by taking the Wick product of $: \omega:_{\mathrm{G}}\left(x_{i}\right), i=1, \ldots, n$, we obtain a Gamma white noise monomial as

$$
\begin{gathered}
: \omega::_{\mathrm{G}}\left(x_{1}\right) \diamond: \omega::_{\mathrm{G}}\left(x_{2}\right) \diamond \cdots \diamond: \omega:_{\mathrm{G}}\left(x_{n}\right)=\left\langle: \omega^{\otimes n}:_{\mathrm{G}}, \delta_{x_{1}} \widehat{\otimes} \cdots \widehat{\otimes} \delta_{x_{n}}\right\rangle \\
=: \omega^{\otimes n}:_{\mathrm{G}}\left(x_{1}, \ldots, x_{n}\right) \in\left(S_{\mathrm{G}}\right)_{-1,-k_{0}}^{-1} .
\end{gathered}
$$

The delta function of Gamma white noise, $\tilde{\delta}_{v}$, where $v \in S^{\prime}$, is defined by

$$
\left\langle\left\langle\tilde{\delta}_{v}, \phi\right\rangle\right\rangle=\phi(v), \quad \phi \in\left(S_{\mathrm{G}}\right)^{1} .
$$

Evidently, $\tilde{\delta}_{v}$ belongs to $\left(S_{\mathrm{G}}\right)^{-1}$ and has the representation:

$$
\tilde{\delta}_{v}=\tilde{\delta}_{v}(\omega)=\sum_{n=0}^{\infty}\left\langle: \omega^{\otimes n}: \mathrm{G}, \frac{1}{n !}: v^{\otimes n}:_{\mathrm{G}}\right\rangle .
$$

Now, we wish to introduce operators of coordinate multiplication, $\omega(x) \cdot$, acting from $\left(S_{\mathrm{G}}\right)^{1}$ into $\left(S_{\mathrm{G}}\right)^{-1}$. To this end, we define first linear operators $\partial_{x}:\left(S_{\mathrm{G}}\right)^{1} \rightarrow$ $\left(S_{\mathrm{G}}\right)^{1}$ and $\partial_{x}^{\dagger}:\left(S_{\mathrm{G}}\right)^{-1} \rightarrow\left(S_{\mathrm{G}}\right)^{-1}$ for each $x \in \operatorname{supp} \sigma$ by

$$
\begin{aligned}
& \partial_{x}\left\langle: \omega^{\otimes n}:_{\mathrm{G}}, f^{(n)}\right\rangle=n\left\langle: \omega^{\otimes(n-1)}:_{\mathrm{G}}, f^{(n)}(x, \cdot)\right\rangle, \\
& \partial_{x}^{\dagger}\left\langle: \omega^{\otimes n}:_{\mathrm{G}}, F^{(n)}\right\rangle=\left\langle: \omega^{\otimes(n+1)}:_{\mathrm{G}}, \delta_{x} \widehat{\otimes} F^{(n)}\right\rangle .
\end{aligned}
$$

It easy to show that these operators are continuous.

Let us preserve the same notations $a^{+}(\xi), a^{0}(\xi), a_{1}^{-}(\xi)$, and $a_{2}^{-}(\xi)$ for the images of the corresponding operators under the unitary $I$. Then, the operators $a^{+}(\xi)$ and 
$a_{1}^{-}(\xi)$ have the integral representation (cf. [12]):

$$
\begin{aligned}
& a_{1}^{-}(\xi)=\int_{\operatorname{supp} \sigma} d \sigma(x) \xi(x) \partial_{x} \\
& a^{+}(\xi)=\int_{\operatorname{supp} \sigma} d \sigma(x) \xi(x) \partial_{x}^{\dagger}
\end{aligned}
$$

Integrals of such type are understood usually in the sense that one applies pointwisely the integrand operator to a test function, then dualizes the result with another test function, and finally integrates the obtained function of $x$ w.r.t. the measure $\sigma$.

As well known, the neutral operator $a^{0}(\xi)$ has the representation

$$
a^{0}(\xi)=\int_{\operatorname{supp} \sigma} d \sigma(x) \xi(x) \partial_{x}^{\dagger} \partial_{x} .
$$

A new point appearing in Gamma analysis is, of course, the second annihilation operator, $a_{2}^{-}(\xi)$, which has now the representation

$$
a_{2}^{-}(\xi)=\int_{\operatorname{supp} \sigma} d \sigma(x) \xi(x) \partial_{x}^{\dagger} \partial_{x} \partial_{x} .
$$

The formula (54) can be verified in a standard way.

Thus, we define the operator $\omega(x) \cdot$ by

$$
\omega(x) \cdot=\partial_{x}^{\dagger}+2 \partial_{x}^{\dagger} \partial_{x}+1+\partial_{x}+\partial_{x}^{\dagger} \partial_{x} \partial_{x}
$$

which acts continuously from $\left(S_{\mathrm{G}}\right)^{1}$ into $\left(S_{\mathrm{G}}\right)^{-1}$. Evidently,

$$
\langle\omega, \xi\rangle \cdot=\int_{\operatorname{supp} \sigma} d \sigma(x) \xi(x) \omega(x) \cdot
$$

By analogy with [13] and [26], the family of operators $(\omega(x) \cdot)_{x \in \operatorname{supp} \sigma}$ can be also called a Gamma field, or a quantum Gamma white noise process.

As will be shown now, the action of all the above operators can be easily represented in terms of the $\mathcal{S}$-transform. Indeed, let $\phi \in\left(S_{\mathrm{G}}\right)^{1}$ and let $U(\theta)=\mathcal{S}[\phi](\theta)$. The formula for $\partial_{x}^{\dagger}$, resp. $a^{+}(\xi)$ is well known (e.g., [11]):

$$
\begin{aligned}
\mathcal{S}\left[\partial_{x}^{\dagger} \phi\right](\theta) & =\theta(x) U(\theta), & & \theta \in S_{\mathbb{C}}, \\
\mathcal{S}\left[a^{+}(\xi) \phi\right](\theta) & =\langle\xi, \theta\rangle U(\theta), & & \theta \in S_{\mathbb{C}}^{\prime}
\end{aligned}
$$

(of course, the formula for $\partial_{x}^{\dagger}$ holds for $\Phi \in\left(S_{\mathrm{G}}\right)^{-1}$ with $\theta$ from a neiborhood of zero in $\left.S_{\mathbb{C}}\right)$. 
Next, analogously to [14], we have that

$$
\begin{aligned}
\mathcal{S}\left[\left(\partial_{x}+2 \partial_{x}^{\dagger} \partial_{x}\right) \phi\right](\theta) & =D_{\delta_{x}(1+2 \theta)} U(\theta), & & \theta \in S_{\mathbb{C}}, \\
\mathcal{S}\left[\left(a_{1}^{-}(\xi)+2 a^{0}(\xi)\right) \phi\right](\theta) & =D_{\xi(1+2 \theta)} U(\theta), & & \theta \in S_{\mathbb{C}}^{\prime},
\end{aligned}
$$

where $D_{v}, v \in S_{\mathbb{C}}^{\prime}$, denotes also the Gâteaux derivatives in direction $v$ of a function defined on $S_{\mathbb{C}}^{\prime}$. Indeed, let $\phi$ be of the form $\phi(\omega)=\left\langle: \omega^{\otimes n}:_{\mathrm{G}}, \varphi^{\otimes n}\right\rangle$, then $U[\phi](\theta)=$ $\left\langle\varphi^{\otimes n}, \theta^{\otimes n}\right\rangle$, and

$$
\begin{gathered}
\left.\frac{d}{d t}\right|_{t=0}\left\langle\varphi^{\otimes n},\left(\theta+t \delta_{x}(1+2 \theta)\right)^{\otimes n}\right\rangle=n \varphi(x)(1+2 \theta(x))\left\langle\varphi^{\otimes(n-1)}, \theta^{\otimes(n-1)}\right\rangle \\
=\mathcal{S}\left[n \varphi(x)\left\langle: \omega^{\otimes(n-1)}:_{\mathrm{G}}, \varphi^{\otimes(n-1)}\right\rangle+2 n \varphi(x)\left\langle: \omega^{\otimes n}:_{\mathrm{G}}, \delta_{x} \widehat{\otimes} \varphi^{\otimes(n-1)}\right\rangle\right](\theta) \\
=\mathcal{S}\left[\left(\partial_{x}+2 \partial_{x}^{\dagger} \partial_{x}\right)\left\langle: \omega^{\otimes n}:_{\mathrm{G}}, \varphi^{\otimes n}\right\rangle\right](\theta), \quad \theta \in S_{\mathbb{C}} .
\end{gathered}
$$

Hence, the formula (56) will be proved if we show that, if $\phi_{m} \rightarrow \phi$ in $\left(S_{\mathrm{G}}\right)^{1}$ as $m \rightarrow \infty$ and $U_{m}(\theta)=\mathcal{S}\left[\phi_{m}\right](\theta)$, then for any fixed $\theta \in S_{\mathbb{C}}$

$$
\lim _{m \rightarrow \infty} D_{\delta_{x}(1+2 \theta)} U_{m}(\theta)=D_{\delta_{x}(1+2 \theta)} U(\theta) .
$$

To this end, we will use the following lemma:

Lemma 3 For each $v \in S_{\mathbb{C}}^{\prime}, D_{v}$ defines a linear continuous operator on $\mathcal{E}_{\min }^{1}\left(S_{\mathbb{C}}^{\prime}\right)$.

Proof. Every function $u \in \mathcal{E}_{\min }^{1}\left(S_{\mathbb{C}}^{\prime}\right)$ can be represented in the form (see e.g. [19])

$$
u(\theta)=\sum_{n=0}^{\infty}\left\langle f^{(n)}, \theta^{\otimes n}\right\rangle, \quad f^{(n)} \in S_{\mathbb{C}}^{\widehat{\otimes} n} .
$$

For each $p \geq 1$ and $k \in \mathbb{N}_{0}$, define the norm

$$
\|u\|_{1, p, k}^{2}=\sum_{n=0}^{\infty}\left|f^{(n)}\right|_{p}^{2}(n !)^{2} 2^{n k},
$$

and let $\mathcal{E}_{p, k}^{1}\left(S_{\mathbb{C}}^{\prime}\right)$ be the closure of $\mathcal{E}_{\min }^{1}\left(S_{\mathbb{C}}^{\prime}\right)$ in this norm. Theorem 3 (see also [19]) implies that

$$
\mathcal{E}_{\min }^{1}\left(S_{\mathbb{C}}^{\prime}\right)=\underset{p, k \rightarrow \infty}{\operatorname{proj} \lim } \mathcal{E}_{p, k}^{1}\left(S_{\mathbb{C}}^{\prime}\right),
$$

i.e., that the sequence of norms (59) is equivalent to (45) Let $v \in S_{-p^{\prime}, \mathbb{C}}$ with $p^{\prime} \geq 1$. Since

$$
D_{v}\left\langle\varphi^{\otimes n}, \theta^{\otimes n}\right\rangle=n\langle\varphi, v\rangle\left\langle\varphi^{\otimes(n-1)}, \theta^{\otimes(n-1)}\right\rangle,
$$

the norm of $D_{v}$ on each $(\mathcal{E})_{p, k}^{1}, p \geq p^{\prime}, k \in \mathbb{N}_{0}$, is not greater than $|v|_{-p} 2^{-k / 2}$, and therefore $D_{v}$ acts continuously on $\mathcal{E}_{\min }^{1}\left(S_{\mathbb{C}}^{\prime}\right)$. 
Now, from Theorem 3 we conclude that the convergence $\phi_{m} \rightarrow \phi$ in $\left(S_{\mathrm{G}}\right)^{1}$ implies the convergence $U_{m} \rightarrow U$ in $\mathcal{E}_{\text {min }}^{1}\left(S_{\mathbb{C}}^{\prime}\right)$, and hence by Lemma $3 D_{v} U_{m} \rightarrow D_{v} U$ in $\mathcal{E}_{\text {min }}^{1}\left(S_{\mathbb{C}}^{\prime}\right)$ for any fixed $v \in S_{\mathbb{C}}^{\prime}$. Therefore, by $(45) D_{v} U_{m}(\theta) \rightarrow D_{v} U(\theta)$ for any fixed $\theta \in S_{\mathbb{C}}^{\prime}$, which evidently implies (58) because $\delta_{x}(1+2 \theta) \in S_{\mathbb{C}}^{\prime}$ for any $\theta \in S_{\mathbb{C}}$. The formula (57) can be proved absolutely analogously to (56) if we take into account that $\xi(1+2 \theta) \in S_{\mathbb{C}}^{\prime}$ for an arbitrary $\theta \in S_{\mathbb{C}}^{\prime}$.

Again analogously to (56), we have

$$
S\left[\partial^{\dagger} \partial_{x} \partial_{x} \phi\right](\theta)=\theta(x) \nabla_{x}^{2} U(\theta), \quad \theta \in S_{\mathbb{C}},
$$

where $\nabla_{x}$ is defined by (48). Then

$$
\mathcal{S}\left[a_{2}^{-}(\xi) \phi\right](\theta)=\left\langle\xi(x) \nabla_{x}^{2} U(\theta), \theta(x)\right\rangle, \quad \theta \in S_{\mathbb{C}}^{\prime},
$$

where $x$ denotes the variable in which the dualization is carried out.

This formula is evidently true for $\phi(\omega)=\left\langle: \omega^{\otimes n}:_{\mathrm{G}}, \varphi^{\otimes n}\right\rangle$ : then $U(\theta)=\left\langle\varphi^{\otimes n}, \theta^{\otimes n}\right\rangle$ and

$$
\begin{aligned}
\left\langle\xi(x) \nabla_{x}^{2}\left\langle\varphi^{\otimes n}, \theta^{\otimes n}\right\rangle, \theta(x)\right\rangle & =\left\langle\xi(x) n(n-1) \varphi(x)^{2}\left\langle\varphi^{\otimes(n-2)}, \theta^{\otimes(n-2)}\right\rangle, \theta(x)\right\rangle \\
& =n(n-1)\left\langle\varphi^{\otimes(n-2)}, \theta^{\otimes(n-2)}\right\rangle\left\langle\xi \varphi^{2}, \theta\right\rangle \\
& =n(n-1)\left\langle\varphi^{\otimes(n-2)} \widehat{\otimes}\left(\xi \varphi^{2}\right), \theta^{\otimes(n-1)}\right\rangle .
\end{aligned}
$$

Hence, it suffices to show that, if $U_{m} \rightarrow U$ in $\mathcal{E}_{\text {min }}^{1}\left(S_{\mathbb{C}}^{\prime}\right)$, then for any fixed $\theta \in S_{\mathbb{C}}^{\prime}$ $\nabla_{x}^{2} U_{m}(\theta) \rightarrow \nabla_{x}^{2} U(\theta)$ in each $S_{p, \mathbb{C}}, p \geq 1$, as a function of $x$.

Representing

$$
U_{m}(\theta)=\sum_{n=0}^{\infty}\left\langle f_{m}^{(n)}, \theta^{\otimes n}\right\rangle \rightarrow U(\theta)=\sum_{n=0}^{\infty}\left\langle f^{(n)}, \theta^{\otimes n}\right\rangle \quad \text { in } \mathcal{E}_{\min }^{1}\left(S_{\mathbb{C}}^{\prime}\right),
$$

we get

$$
\begin{aligned}
\nabla_{x}^{2} U_{m}(\theta) & =\sum_{n=0}^{\infty} \nabla_{x}^{2}\left\langle f_{m}^{(n)}, \theta^{\otimes n}\right\rangle \\
& =\sum_{n=2}^{\infty} n(n-1)\left\langle f_{m}^{(n)}(x, x, \cdot), \theta^{\otimes(n-2)}\right\rangle .
\end{aligned}
$$

(61) yields that $f_{m}^{(n)} \rightarrow f^{(n)}$ in $S_{\mathbb{C}}^{\widehat{\otimes} n}$, and therefore

$$
\left\langle f_{m}^{(n)}(x, x, \cdot), \theta^{\otimes(n-2)}\right\rangle \rightarrow\left\langle f^{(n)}(x, x, \cdot), \theta^{\otimes(n-2)}\right\rangle \quad \text { in } S_{\mathbb{C}}
$$

Moreover, upon (5) we have, for an arbitrary $v \in S_{-p, \mathbb{C}}$,

$$
\begin{aligned}
\mid\langle v(x) & \left.,\left\langle f_{m}^{(n)}(x, x, \cdot), \theta^{\otimes(n-2)}\right\rangle\right\rangle \mid \\
& =\left|\left\langle f_{m}^{(n)}\left(x_{1}, x_{1}, x_{2}, \ldots, x_{n-1}\right), v\left(x_{1}\right) \theta^{\otimes(n-2)}\left(x_{2}, \ldots, x_{n-1}\right)\right\rangle\right| \\
& \leq|v|_{-p}|\theta|_{-p}^{n-2} C_{p}\left|f_{m}^{(n)}\right|_{p},
\end{aligned}
$$


whence taking to notice that $S_{p, \mathbb{C}}$ can be thought of as the dual of $S_{-p, \mathbb{C}}$, we get

$$
\left|\left\langle f_{m}^{(n)}(x, x, \cdot), \theta^{\otimes(n-2)}\right\rangle\right|_{p} \leq C_{p}|\theta|_{-p}^{n-2}\left|f_{m}^{(n)}\right|_{p} .
$$

Estimating

$$
\begin{aligned}
& \sum_{n=2}^{\infty} n(n-1)\left|\left\langle f_{m}^{(n)}(x, x, \cdot), \theta^{\otimes(n-2)}\right\rangle\right|_{p} \\
& \quad \leq C_{p} \sum_{n=2}^{\infty} n(n-1)|\theta|_{-p}^{n-2}\left|f_{m}^{(n)}\right|_{p} \\
& \quad \leq C_{p} \sum_{n=2}^{\infty}((n-2) !)^{-1}|\theta|_{-p}^{n-2}\left|f_{m}^{(n)}\right|_{p} n ! \\
& \leq C_{p}\left(\sum_{n=0}^{\infty}(n !)^{-2}|\theta|_{-p}^{2 n}\right)^{1 / 2}\left\|U_{m}\right\| \|_{1, p, 0},
\end{aligned}
$$

we obtain the desired statement.

Thus, we have proved the following theorem.

Theorem 5 Let $\phi \in\left(S_{\mathrm{G}}\right)^{1}$, then the action of the operators $\omega(x) \cdot, x \in \operatorname{supp} \sigma$, and $\langle\omega, \xi\rangle \cdot \xi \in S$, can be represented in terms of the $\mathcal{S}$-transform as follows:

$$
\begin{aligned}
\mathcal{S}[\omega(x) \cdot \phi](\theta) & =(\theta(x)+1) U(\theta)+D_{\delta_{x}(1+2 \theta)} U(\theta)+\theta(x) \nabla_{x}^{2} U(\theta), & & \theta \in S_{\mathbb{C}}, \\
\mathcal{S}[\langle\omega, \xi\rangle \cdot \phi](\theta) & =\langle\xi, \theta+1\rangle U(\theta)+D_{\xi(1+2 \theta)} U(\theta)+\left\langle\xi(x) \nabla_{x}^{2} U(\theta), \theta(x)\right\rangle, & & \theta \in S_{\mathbb{C}}^{\prime},
\end{aligned}
$$

where $U(\theta)=\mathcal{S}[\phi](\theta)$.

\section{Standard annihilation operator on Gamma space}

In this section, we will study the standard annihilation operators $\partial_{x}$ and $a_{1}^{-}(\xi)$.

First, we note that

$$
\sup _{x \in \operatorname{supp} \sigma}\left|\delta_{x}\right|_{-p}=\|\delta\|_{p, \infty}<\infty .
$$

Therefore, for each $x \in \operatorname{supp} \sigma, \partial_{x}$ can be extended to a continuous operator on $\left(S_{\mathrm{G}}\right)_{p, k}^{1}, p \geq 1, k \geq k_{0}$, with norm $\leq\|\delta\|_{p, \infty} 2^{-k / 2}$.

It follows from the proof of Theorem 4 that $\partial_{x}$ is nothing but the $\alpha$-gradient with $\alpha(\varphi)=\frac{\varphi}{\varphi+1}$, and on the total set in $\left(S_{\mathrm{G}}\right)_{p, k}^{1}$ consisting of the Wick exponentials $: e^{\langle\cdot, \varphi\rangle}:_{\mathrm{G}}$ with $|\varphi|_{p}<2^{-k / 2}$ we have

$$
\partial_{x}: e^{\langle\omega, \varphi\rangle}:_{\mathrm{G}}=\nabla_{x}^{\alpha}: e^{\langle\omega, \varphi\rangle}:_{\mathrm{G}}=\varphi(x): e^{\langle\omega, \varphi\rangle}:_{\mathrm{G}} .
$$


Note that

$$
\alpha^{-1}(\varphi)=\frac{\varphi}{1-\varphi}=\sum_{n=1}^{\infty} \varphi^{n}
$$

and hence in virtue of $(5)$

$$
\left|\alpha^{-1}(\varphi)\right|_{p} \leq \frac{|\varphi|_{p}}{1-C_{p}|\varphi|_{p}} \quad \text { if }|\varphi|_{p}<C_{p}
$$

Choosing $\varphi \in S$ such that

$$
|\varphi|_{p}<\frac{2^{-k / 2}}{1+C_{p} 2^{-k / 2}}
$$

we get that $\left|\alpha^{-1}(\varphi)\right|_{p}<2^{-k / 2}$, and then upon (49)-(51)

$$
\begin{aligned}
\partial_{x} e_{\mathrm{G}}(\varphi ; \omega) & =\partial_{x}: e^{\left\langle\omega, \alpha^{-1}(\varphi)\right\rangle}:_{\mathrm{G}} \\
& =\frac{\varphi(x)}{1-\varphi(x)} e_{\mathrm{G}}(\varphi ; \omega) \\
& =\sum_{n=1}^{\infty} \varphi(x)^{n} e_{\mathrm{G}}(\varphi ; \omega) .
\end{aligned}
$$

Since

$$
\alpha(\varphi)=\frac{\varphi}{\varphi+1}=\sum_{n=1}^{\infty}(-1)^{n+1} \varphi^{n},
$$

the functions $e_{\mathrm{G}}(\varphi ; \omega)$ also constitute a total set in $\left(S_{\mathrm{G}}\right)_{p, k}^{1}$, and therefore, at least formally, we can write down

$$
\partial_{x}=\alpha^{-1}\left(\nabla_{x}\right)=\frac{\nabla_{x}}{1-\nabla_{x}}=\sum_{n=1}^{\infty} \nabla_{x}^{n} .
$$

Let $\mathcal{E}_{p, k}^{1}\left(S^{\prime}\right)$ denote the Hilbert space constructed in the same way as $\mathcal{E}_{p, k}^{1}\left(S_{\mathbb{C}}^{\prime}\right)$ (see the proof of Lemma 3) but only starting from $\mathcal{E}_{\text {min }}^{1}\left(S^{\prime}\right)$. Choosing an arbitrary $\tilde{p} \geq 1$ and $\tilde{k} \in \mathbb{N}_{0}$ such that $2^{\tilde{k} / 2}>\|\delta\|_{\tilde{p}, \infty}$, we get that each $\nabla_{x}$ is a continuous operator on $\mathcal{E}_{\tilde{p}, \tilde{k}}^{1}\left(S^{\prime}\right)$ with norm less than one. Hence, the series $\sum_{n=1}^{\infty} \nabla_{x}^{n}$ converges in operator norm. Let now $p \geq 1$ and $k \geq k_{0}$ be chosen so that the space $\left(S_{\mathrm{G}}\right)_{p, k}^{1}$ is topologically embedded into $\mathcal{E}_{\tilde{p}, \tilde{k}}^{1}\left(S^{\prime}\right)$. Then, the above functions $e_{\mathrm{G}}(\varphi ; \omega)$ constitute also a total set in $\mathcal{E}_{\tilde{p}, \tilde{k}}^{1}\left(S^{\prime}\right)$, which implies the equality (62) on each space $\mathcal{E}_{\tilde{p}, \tilde{k}}^{1}\left(S^{\prime}\right)$, and therefore on $\mathcal{E}_{\min }^{1}\left(S^{\prime}\right)$.

In the same way, one can derive the inverse representation of (62)

$$
\nabla_{x}=\alpha\left(\partial_{x}\right)=\frac{\partial_{x}}{\partial_{x}+1}=\sum_{n=1}^{\infty}(-1)^{n+1} \partial_{x}^{n} .
$$


As a corollary of (62) or (63), we have the commutation of arbitrary $\nabla_{x_{1}}$ and $\partial_{x_{2}}$ on $\left(S_{\mathrm{G}}\right)^{1}$.

Now, we will show that analogously to the one-dimensional case (see e.g. [28]) the operators $\partial_{x}$ and $a_{1}^{-}(\xi)$ have a representation as an integral w.r.t. a difference operator.

Theorem 6 For an arbitrary $\phi \in\left(S_{\mathrm{G}}\right)^{1}$, we have

$$
\begin{aligned}
\left(\partial_{x} \phi\right)(\omega) & =\int_{0}^{\infty}\left(\phi\left(\omega+s \delta_{x}\right)-\phi(\omega)\right) e^{-s} d s \\
& =\int_{0}^{\infty} \phi\left(\omega+s \delta_{x}\right) e^{-s} d s-\phi(\omega), \quad x \in \operatorname{supp} \sigma \\
\left(a_{1}^{-}(\xi) \phi\right)(\omega) & =\int_{\operatorname{supp} \sigma} \int_{0}^{\infty} \xi(x)\left(\phi\left(\omega+s \delta_{x}\right)-\phi(\omega)\right) e^{-s} d s d \sigma(x), \quad \xi \in S .
\end{aligned}
$$

Proof. Let $\theta \in S_{-p, \mathbb{C}}$, then for any $u \in \mathcal{E}_{\min }^{1}\left(S_{\mathbb{C}}^{\prime}\right)$,

$$
\begin{aligned}
\left|\int_{0}^{\infty} u\left(\theta+s \delta_{x}\right) e^{-s} d s\right| & \leq|u|_{1, p, k} \int_{0}^{\infty} \exp \left[\frac{1}{k}\left|\theta+s \delta_{x}\right|_{-p}\right] e^{-s} d s \\
& \leq|u|_{1, p, k} \exp \left[\frac{1}{k}|\theta|_{-p}\right] \int_{0}^{\infty} \exp \left[-s\left(1-\frac{1}{k} \|\left.\delta\right|_{p, \infty}\right)\right] d s \\
& =|u|_{1, p, k} \exp \left[\frac{1}{k}|\theta|_{-p}\right] \frac{1}{1-\frac{1}{k}\|\delta\|_{p, \infty}}, \quad k>\|\delta\|_{p, \infty}
\end{aligned}
$$

where the norm $|\cdot|_{1, p, k}$ is defined by (45). Hence

$$
\left|\int_{0}^{\infty} u\left(\cdot+s \delta_{x}\right) e^{-s} d s\right|_{1, p, k} \leq|u|_{1, p, k} \frac{1}{1-\frac{1}{k}\|\delta\|_{p, \infty}} .
$$

Therefore, by Theorem 4 the operator $A_{x}$ defined by the right hand side of (64) determines a linear continuous operator on $\left(S_{\mathrm{G}}\right)^{1}$, i.e., for arbitrary $p \geq 1$ and $k \geq k_{0}$, there are $p^{\prime} \geq p$ and $k^{\prime} \geq k$ such that $A_{x}$ acts continuously from $\left(S_{\mathrm{G}}\right)_{p^{\prime}, k^{\prime}}^{1}$ into $\left(S_{\mathrm{G}}\right)_{p, k}^{1}$. On the other hand, $\partial_{x}$ acts also continuously on $\left(S_{\mathrm{G}}\right)^{1}$, and in particular on each $\left(S_{\mathrm{G}}\right)_{p^{\prime}, k^{\prime}}^{1}$. Hence, it suffices to prove the equality $(64)$ on a total set in $\left(S_{\mathrm{G}}\right)_{p^{\prime}, k^{\prime}}^{1}$. As such a set we take the functions $: e^{\langle\cdot, \varphi\rangle}:_{\mathrm{G}}$ with $|\varphi|_{p^{\prime}}<2^{-k^{\prime} / 2}$. Then,

$$
\partial_{x}: e^{\langle\omega, \varphi\rangle}:_{\mathrm{G}}=\varphi(x): e^{\langle\omega, \varphi\rangle}:_{\mathrm{G}},
$$


and from (36) we derive that

$$
\begin{aligned}
\int_{0}^{\infty}: e^{\left\langle\omega+s \delta_{x}, \varphi\right\rangle}:_{\mathrm{G}} e^{-s} d s & =\int_{0}^{\infty} \exp \left[\left\langle\omega+s \delta_{x}, \frac{\varphi}{\varphi+1}\right\rangle-\langle\log (1+\varphi)\rangle\right] e^{-s} d s \\
& =\exp \left[\left\langle\omega, \frac{\varphi}{\varphi+1}\right\rangle-\langle\log (1+\varphi)\rangle\right] \int_{0}^{\infty} \exp \left[\frac{s \varphi(x)}{\varphi(x)+1}-s\right] d s \\
& =: e^{\langle\omega, \varphi\rangle}:_{\mathrm{G}} \int_{0}^{\infty} \exp \left[\frac{-s}{\varphi(x)+1}\right] d s \\
& =: e^{\langle\omega, \varphi\rangle}:_{\mathrm{G}}(\varphi(x)+1)
\end{aligned}
$$

which proves (64). The formula (65) can be proved absolutely analogously.

Remark 9. Let $\mu_{\mathrm{CP}}$ be a measure of compound Poisson (CP) white noise on $S^{\prime}$ with Lévy measure $\sigma \nu$, i.e., the Laplace transform of $\mu_{\mathrm{CP}}$ is given by

$$
\int_{S^{\prime}} \exp [\langle\omega, \varphi\rangle] d \mu_{\mathrm{CP}}(\omega)=\exp \left[\int_{\mathbb{R}^{d+1}}\left(e^{s \varphi(x)}-1\right) d \sigma(x) d \nu(s)\right] .
$$

Then, one can study CP analysis by using an isomorphism between the usual Fock space over $L^{2}\left(\mathbb{R}^{d+1}, d \sigma d \nu\right)$ and the $L^{2}$-space of $\mathrm{CP}$ white noise $\left(L_{\mathrm{CP}}^{2}\right)=$ $L^{2}\left(S^{\prime}, \mathcal{B}\left(S^{\prime}\right), d \mu_{\mathrm{CP}}\right)[26,22,21]$.

The CP-field operators $a(\xi), \xi \in S$, have in the Fock space the following representation:

$$
a(\xi)=a^{+}(\xi \otimes \mathrm{id})+a^{0}(\xi \otimes \mathrm{id})+\int_{\mathbb{R}^{d}} \xi(x) d \sigma(x) \int_{\mathbb{R}} s d \nu(s) \mathrm{id}+a^{-}(\xi \otimes \mathrm{id}),
$$

where $\xi \otimes \mathrm{id}=(\xi \otimes \mathrm{id})(x, s)=\xi(x) s$ and $a^{+}, a^{0}, a^{-}$are the standard creation, neutral, and annihilation operators, respectively.

(The image of) the annihilation operator $a^{-}(\xi \otimes \mathrm{id})$ acts in the following way in the space $\left(L_{\mathrm{CP}}^{2}\right)$ :

$$
\left(a^{-}(\xi \otimes \mathrm{id}) \phi\right)(\omega)=\int_{\mathbb{R}^{d+1}} \xi(x) s\left(\phi\left(\omega+s \delta_{x}\right)-\phi(\omega)\right) d \sigma(x) d \nu(s) .
$$

In case of the Gamma measure, $d \nu(s)=\frac{e^{-s}}{s} d s$ (see (25)), and therefore the formula (67) takes the form

$$
\left(a^{-}(\xi \otimes \mathrm{id}) \phi\right)(\omega)=\int_{\mathbb{R}^{d+1}} \xi(x)\left(\phi\left(\omega+s \delta_{x}\right)-\phi(\omega)\right) e^{-s} d \sigma(x) d s .
$$

Hence, $a^{-}(\xi \otimes \mathrm{id})$ is just the operator $a_{1}^{-}(\xi)$ under consideration in this paper.

As a consequence of this, we can write down the explicit action of the adjoint operator of $a_{1}^{-}(\xi)$ in $\left(L_{\mathrm{G}}^{2}\right)$, denoted by $a_{1}^{+}(\xi)$, which corresponds to the operator $a^{+}(\xi \otimes \mathrm{id})$, see $[31,22]$. Thus, for an arbitrary $\omega \in S^{\prime}$ of the form (26)

$$
a_{1}^{+}(\xi) \phi(\omega)=\left\langle\omega(x), \xi(x) \phi\left(\omega-s_{x} \delta_{x}\right)\right\rangle-\langle\xi\rangle \phi(\omega), \quad \phi \in \operatorname{Dom} a^{+}(\xi) .
$$




\section{Creation, neutral, and Gamma annihilation operators on the Gamma space}

In this section, we will obtain the explicit formulas for the operators $a^{+}(\xi), a^{0}(\xi)$, and $a_{2}^{-}(\xi)$ acting on $\left(L_{\mathrm{G}}^{2}\right)$ by using the formula (55), which expresses the operator of coordinate multiplication via $\partial_{x}$ and $\partial_{x}^{\dagger}$ and Theorem 6 .

Let us fix arbitrary $\tilde{p} \geq 1$ and $\tilde{k} \geq k_{0}$, then each $\partial_{x}$ can be extended to a continuous operator on $\left(S_{\mathrm{G}}\right)_{\tilde{p}, \tilde{k}}^{1}$ and $\partial_{x}^{\dagger}$ and $\omega(x)$. to continuous operators from $\left(S_{\mathrm{G}}\right)_{\tilde{p}, \tilde{k}}^{1}$ into $\left(S_{\mathrm{G}}\right)_{-\tilde{p},-\tilde{k}}^{-1}$. Choose now $p \geq \tilde{p}$ and $k \in \mathbb{N}_{0}$ so that the space $\mathcal{E}_{p, k}^{1}\left(S^{\prime}\right)$ is topologically embedded into $\left(S_{\mathrm{G}}\right)_{\tilde{p}, \tilde{k}}^{1}$. The restrictions of $\partial_{x}, \partial_{x}^{\dagger}$, and $\omega(x) \cdot$ to $\mathcal{E}_{p, k}^{1}\left(S^{\prime}\right)$ are continuous operators from this space into $\left(S_{\mathrm{G}}\right)_{\tilde{p}, \tilde{k}}^{1}$ and $\left(S_{\mathrm{G}}\right)_{-\tilde{p},-\tilde{k}}^{-1}$, respectively. We rewrite the formula (55) as follows:

$$
\omega(x) \cdot=\partial_{x}^{\dagger}\left(\partial_{x}+1\right)^{2}+\left(\partial_{x}+1\right) .
$$

Due to the (proof of) Theorem 6, we have for an arbitrary $\phi \in \mathcal{E}_{p, k}^{1}\left(S^{\prime}\right)$ :

$$
\left(\partial_{x}+1\right) \phi(\omega)=\int_{0}^{\infty} \phi\left(\omega+s \delta_{x}\right) e^{-s} d s
$$

and hence on the total set in $\mathcal{E}_{p, k}^{1}\left(S^{\prime}\right)$ consisting of the functions $e^{\langle\omega, \varphi\rangle}$ with $|\varphi|_{p}<$ $2^{-k / 2}$ we have analogously to $(66)$ :

$$
\left(\partial_{x}+1\right) e^{\langle\omega, \varphi\rangle}=(1-\varphi(x))^{-1} e^{\langle\omega, \varphi\rangle} .
$$

Therefore, by (68),

$$
\omega(x) \cdot e^{\langle\omega, \varphi\rangle}=(1-\varphi(x))^{-2} \partial_{x}^{\dagger} e^{\langle\omega, \varphi\rangle}+(1-\varphi(x))^{-1} e^{\langle\omega, \varphi\rangle},
$$

which yields

$$
\begin{aligned}
\partial_{x}^{+} e^{\langle\omega, \varphi\rangle} & =\omega(x) \cdot(\varphi(x)-1)^{2} e^{\langle\omega, \varphi\rangle}+(\varphi(x)-1) e^{\langle\omega, \varphi\rangle} \\
& =\omega(x) \cdot\left(\nabla_{x}-1\right)^{2} e^{\langle\omega, \varphi\rangle}+\left(\nabla_{x}-1\right) e^{\langle\omega, \varphi\rangle} .
\end{aligned}
$$

Since $\nabla_{x}$ is a continuous operator on $\mathcal{E}_{p, k}^{1}\left(S^{\prime}\right)$, this immediately implies the central lemma of this section:

Lemma 4 We have on $\left(S_{\mathrm{G}}\right)^{1}$ :

$$
\partial_{x}^{\dagger}=\omega(x) \cdot\left(\nabla_{x}-1\right)^{2}+\left(\nabla_{x}-1\right)
$$


Now, by using (53) and Lemma 4, we can calculate, at least formally, the action of $a^{+}(\xi)$ :

$$
\begin{aligned}
a^{+}(\xi) & =\int_{\operatorname{supp} \sigma} d \sigma(x) \xi(x)\left(\omega(x) \cdot\left(\nabla_{x}-1\right)^{2}+\left(\nabla_{x}-1\right)\right) \\
& =\left\langle\omega(x), \xi(x)\left(\nabla_{x}-1\right)^{2}\right\rangle+\left\langle\xi(x), \nabla_{x}-1\right\rangle .
\end{aligned}
$$

Theorem 7 For any $\phi \in\left(S_{\mathrm{G}}\right)^{1}$,

$$
a^{+}(\xi) \phi(\omega)=\left\langle\omega(x), \xi(x)\left(\nabla_{x}-1\right)^{2} \phi(\omega)\right\rangle+\left(D_{\xi}-\langle\xi\rangle\right) \phi(\omega), \quad \omega \in S^{\prime},
$$

where $x$ denotes the variable in which the dualization is carried out.

Proof. Analogously to the above, we will consider $a^{+}(\xi)$ as a continuous operator from $\mathcal{E}_{p, k}^{1}\left(S^{\prime}\right)$ into $\left(S_{\mathrm{G}}\right)_{\tilde{p}, \tilde{k}}^{1}$. Take again $\phi(\omega)=e^{\langle\omega, \varphi\rangle}$ with $|\varphi|_{p}<2^{-k / 2}$. Then, using (53) and Lemma 4 , we have for an arbitrary $\psi \in\left(S_{\mathrm{G}}\right)^{1}$ :

$$
\begin{aligned}
\left\langle\left\langle a^{+}(\xi) e^{\langle\omega, \varphi\rangle}, \psi(\omega)\right\rangle\right\rangle & \\
= & \int_{\operatorname{supp} \sigma} d \sigma(x) \xi(x)\left\langle\left\langle\left(\omega(x) \cdot\left(\nabla_{x}-1\right)^{2}+\left(\nabla_{x}-1\right)\right) e^{\langle\omega, \varphi\rangle}, \psi(\omega)\right\rangle\right\rangle \\
= & \int_{\operatorname{supp} \sigma} d \sigma(x) \xi(x)(\varphi(x)-1)^{2}\left\langle\left\langle\omega(x) \cdot e^{\langle\omega, \varphi\rangle}, \psi(\omega)\right\rangle\right\rangle \\
& +\int_{\operatorname{supp} \sigma} d \sigma(x) \xi(x)(\varphi(x)-1)\left\langle\left\langle e^{\langle\omega, \varphi\rangle}, \psi(\omega)\right\rangle\right\rangle \\
= & \left\langle\left\langle\left\langle\omega, \xi(\varphi-1)^{2}\right\rangle e^{\langle\omega, \varphi\rangle}, \psi(\omega)\right\rangle\right\rangle+\langle\xi, \varphi-1\rangle\left\langle\left\langle e^{\langle\omega, \varphi\rangle}, \psi(\omega)\right\rangle\right\rangle \\
= & \left\langle\left\langle\left\langle\omega(x), \xi(x)(\varphi(x)-1)^{2} e^{\langle\omega, \varphi\rangle}\right\rangle+\langle\xi, \varphi-1\rangle e^{\langle\omega, \varphi\rangle}, \psi(\omega)\right\rangle\right\rangle .
\end{aligned}
$$

Therefore,

$$
a^{+}(\xi) e^{\langle\omega, \varphi\rangle}=\left\langle\omega(x), \xi(x)(\varphi(x)-1)^{2} e^{\langle\omega, \varphi\rangle}\right\rangle+\langle\xi, \varphi-1\rangle e^{\langle\omega, \varphi\rangle},
$$

where the equality in understood as that in $\left(S_{\mathrm{G}}\right)^{-1}$. But the right hand side of $(69)$ considered as a function of $\omega$ belongs to $\mathcal{E}_{p, k}^{1}\left(S^{\prime}\right)$, which follows from the representation

$$
\left\langle\omega(x), \xi(x)(\varphi(x)-1)^{2} e^{\langle\omega, \varphi\rangle}\right\rangle=\sum_{n=1}^{\infty}\left\langle\omega^{\otimes n}, \frac{1}{(n-1) !} \varphi^{\otimes(n-1)} \widehat{\otimes}\left(\xi(\varphi-1)^{2}\right)\right\rangle .
$$

Therefore, (69) holds for each $\omega \in S_{-p}$.

It remains only to note that $D_{\xi}$ is a continuous operator on $\mathcal{E}_{p, k}^{1}\left(S^{\prime}\right)$ and for any fixed $\omega \in S_{-p}\left(\nabla_{x}-1\right)^{2} \phi_{m}(\omega) \rightarrow\left(\nabla_{x}-1\right)^{2} \phi(\omega)$ in $S_{p}$ as a function of $x$ if $\phi_{m} \rightarrow \phi$ in $\mathcal{E}_{p, k}^{1}\left(S^{\prime}\right)$, the latter being proved in the same way as the formula (60).

We proceed to consider the neutral operator $a^{0}(\xi)$ on the Gamma space. 
Lemma 5 We have on $\left(S_{\mathrm{G}}\right)^{1}$ :

$$
\partial_{x}^{\dagger} \partial_{x}=\omega(x) \cdot \nabla_{x}\left(1-\nabla_{x}\right)-\nabla_{x}, \quad x \in \operatorname{supp} \sigma .
$$

Proof. Using Lemma 4 and its proof, we get

$$
\begin{gathered}
\partial_{x}^{\dagger} \partial_{x} e^{\langle\omega, \varphi\rangle}=\left(\omega(x) \cdot\left(\nabla_{x}-1\right)^{2}+\left(\nabla_{x}-1\right)\right) \partial_{x} e^{\langle\omega, \varphi\rangle} \\
=\left(\omega(x) \cdot\left(\nabla_{x}-1\right)^{2}+\left(\nabla_{x}-1\right)\right) \frac{\varphi(x)}{1-\varphi(x)} e^{\langle\omega, \varphi\rangle} \\
=\left(\omega(x) \cdot \frac{(\varphi(x)-1)^{2} \varphi(x)}{1-\varphi(x)}+\frac{(\varphi(x)-1) \varphi(x)}{1-\varphi(x)}\right) e^{\langle\omega, \varphi\rangle} \\
=(\omega(x) \cdot(1-\varphi(x)) \varphi(x)-\varphi(x)) e^{\langle\omega, \varphi\rangle} \\
=\left(\omega(x) \cdot\left(1-\nabla_{x}\right) \nabla_{x}-\nabla_{x}\right) e^{\langle\omega, \varphi\rangle} .
\end{gathered}
$$

Again, due to the continuity of $\nabla_{x}$ on $\mathcal{E}_{p, k}^{1}\left(S^{\prime}\right)$, we obtain the lemma.

Since formally

$$
\begin{aligned}
a^{0}(\xi) & =\int_{\operatorname{supp} \sigma} d \sigma(x) \partial_{x}^{\dagger} \partial_{x} \\
& =\int_{\operatorname{supp} \sigma} d \sigma(x) \xi(x)\left(\omega(x) \cdot \nabla_{x}\left(1-\nabla_{x}\right)-\nabla_{x}\right) \\
& =\left\langle\omega(x), \xi(x) \nabla_{x}\left(1-\nabla_{x}\right)\right\rangle+\left\langle\xi(x), \nabla_{x}\right\rangle
\end{aligned}
$$

we come to the following theorem, whose proof is analogous to that of Theorem 7 .

Theorem 8 For any $\phi \in\left(S_{\mathrm{G}}\right)^{1}$,

$$
a^{0}(\xi) \phi(\omega)=\left\langle\omega(x), \xi(x) \nabla_{x}\left(1-\nabla_{x}\right) \phi(\omega)\right\rangle-D_{\xi} \phi(\omega), \quad \omega \in S^{\prime} .
$$

Finally, we will shortly consider the Gamma annihilation operator. Analogously to Lemma 5, we get

Lemma 6 We have on $\left(S_{\mathrm{G}}\right)^{1}$

$$
\begin{aligned}
\partial_{x}^{\dagger} \partial_{x}^{2} & =\omega(x) \cdot \nabla_{x}^{2}-\partial_{x} \nabla_{x} \\
& =\omega(x) \cdot \nabla_{x}^{2}-\nabla_{x} \partial_{x} .
\end{aligned}
$$

Theorem 9 For any $\phi \in\left(S_{\mathrm{G}}\right)^{1}$

$$
\begin{aligned}
a_{2}^{-}(\xi) \phi(\omega)= & \left\langle\omega(x), \xi(x) \nabla_{x}^{2} \phi(\omega)\right\rangle+D_{\xi} \phi(\omega) \\
& -\int_{\operatorname{supp} \sigma} \int_{0}^{\infty} \xi(x) \phi\left(\omega+s \delta_{x}\right) e^{-s} d s d \sigma(x)-\langle\xi\rangle \phi(\omega) \\
= & \left\langle\omega(x), \xi(x) \nabla_{x}^{2} \phi(\omega)\right\rangle+D_{\xi} \phi(\omega) \\
& -\int_{\operatorname{supp} \sigma} \int_{0}^{\infty} \xi(x) \nabla_{x} \phi\left(\omega+s \delta_{x}\right) e^{-s} d s d \sigma(x) .
\end{aligned}
$$


Proof. The formula (70) follows directly from the equality

$$
a_{2}^{-}(\xi)=\langle\omega, \xi\rangle \cdot-a^{+}(\xi)-2 a^{0}(\xi)-\langle\xi\rangle \mathrm{id}-a_{1}^{-}(\xi)
$$

and from Theorems 6-8. The equivalent formula (71) is obtained from Lemma 6 and Theorem 6 just as above. The only new point here is to prove that

$$
\int_{\operatorname{supp} \sigma} d \sigma(x) \xi(x) \partial_{x} \nabla_{x} \phi_{m}(\omega) \rightarrow \int_{\operatorname{supp} \sigma} d \sigma(x) \xi(x) \partial_{x} \nabla_{x} \phi(\omega), \quad \omega \in S^{\prime},
$$

if $\phi_{m} \rightarrow \phi$ in $\left(S_{\mathrm{G}}\right)^{1}$ as $m \rightarrow \infty$. Bur for each $x \in \operatorname{supp} \sigma$ the norm of $\nabla_{x}$ on the space $\mathcal{E}_{p, k}^{1}\left(S^{\prime}\right)$ does not exceed $\|\delta\|_{p, \infty} 2^{-k / 2}<1$ under an appropriate choice of $k$, so that each $\nabla_{x} \partial_{x}=\sum_{n=2}^{\infty} \nabla_{x}^{n}$ (see $\left.(62)\right)$ is a continuous operator on $\mathcal{E}_{p, k}^{1}\left(S^{\prime}\right)$ whose norm is bounded by a constant uniformly in $x$. Therefore, since $\xi \in S \subset L^{1}(\operatorname{supp} \sigma, d \sigma)$, $\int_{\operatorname{supp} \sigma} d \sigma(x) \xi(x) \nabla_{x} \partial_{x}$ is a continuous operator on $\mathcal{E}_{p, k}^{1}$, which implies (72).

\section{ACKNOWLEDGMENTS}

We would like to thank J. L. Silva for useful discussions. The authors were partially supported by the SFB 256, Bonn University. Yu.K. acknowledges partial financial support through the INTAS-Project Nr. 97-0378.

\section{References}

[1] S. Albeverio, Yu. L. Daletsky, Yu. G. Kondratiev, and L. Streit, Non-Gaussian infinite dimensional analysis, J. Func. Anal. 136 (1996), 1-42.

[2] Yu. M. Berezansky, Expansion in Eigenfunctions of Selfadjoint Operators, Amer. Math. Soc., Providence, R. I., 1968.

[3] Yu. M. Berezansky, Spectral approach to white noise analysis, "Bielefeld Encounters in Mathematical Physics VIII," pp. 131-140, World Scientific, Singapore, New Jersey, London, Hong-Kong, 1993.

[4] Yu. M. Berezansky, Commutative Jacobi fields in Fock space, Integr. Equ. Oper. Theory 30 (1998), 163-190.

[5] Yu. M. Berezansky and Yu. G. Kondratiev, "Spectral Methods in Infinite Dimensional Analysis," Kluwer Acad. Publ., Dordrecht, Boston, London, 1994.

[6] Yu. M. Berezansky, V. O. Livinsy, and E. W. Lytvynov, A generalization of Gaussian white noise analysis, Meth. Func. Anal. and Topol. 1 (1995), 28-55. 
[7] T. S. Chihara, "An Introduction to Orthogonal Polynomials," Gordon and Breach Sci. Pbl., New York, London, Paris, 1978.

[8] A. Dermoune, Distributions sur l'espace de P. Lévy et calcul stochastique, Ann. Inst. H. Poincaré Probab. Statist. 26(1) (1990), 101-119.

[9] I. M. Gel'fand, M. I. Graev, and A. M. Vershik, Representations of the group of diffeomorphisms, Russian Math. Surveys 30(6) (1975), 3-50.

[10] I. M. Gel'fand and N. Ya. Vilenkin, "Generalized Functions," Vol. IV, Academic Press, New York, London, 1964.

[11] T. Hida, H.-H. Kuo, J. Potthoff, and L. Streit, "White Noise: An Infinite Dimensional Calculus," Kluwer Acad. Publ., Dordrecht, Boston, London, 1993.

[12] T. Hida, N. Obata, and K. Saitô, Infinite dimensional rotations and Laplacians in terms of white noise calculus, Nagoya Math. J. 128 (1992), 65-93.

[13] Z. Huang, Quantum white noise - White noise approach to quantum stochastic calculus, Nagoya Math. J. 29 (1993), 23-42.

[14] Y. Ito and I. Kubo, Calculus on Gaussian and Poisson white noises, Nagoya Math. J. 111 (1988), 41-84.

[15] O. Kallenberg, "Random Measures," Akedemie-Verlag, Berlin, third edition, 1983.

[16] O. Kallenberg, "Foundations of Modern Probability," Springer-Verlag, New York, Berlin, Heidelberg, 1997.

[17] J. Kerstan, K. Matthes, and J. Mecke, "Infinite Divisible Point Processes, Akademie-Verlag, Berlin, 1978.

[18] J. F. C. Kingman, "Poisson Processes," Clarendon Press, Oxford, 1993.

[19] Yu. G. Kondratiev, P. Leukert, and L. Streit, Wick calculus in Gaussian analysis, Acta Appl. Math. 44 (1996), 269-294.

[20] Yu. G. Kondratiev, J. L. Silva, and L. Streit, Generalized Appell systems, Meth. Func. Anal. and Topol. 3 (1997), no. 3, 28-61.

[21] Yu. G. Kondratiev, J. L. Silva, and L. Streit, Differential geometry on compound Poisson space, Meth. Funct. Anal. and Topol. 4 (1998), no. 1, 32-58.

[22] Yu. G. Kondratiev, J. L. Silva, L. Streit, and G. F. Us, Analysis on Poisson and Gamma spaces, Infinite Dimensional Analysis, Quantum Probability and Related Topics 1 (1998), 91-117. 
[23] Yu. G. Kondratiev, L. Streit, W. Westerkamp, and J. Yan, Generalized functions in infinite dimensional analysis, to appear in Hiroshima Math. J.

[24] E. W. Lytvynov, Multiple Wiener integrals and non-Gaussian white noises: a Jacobi field approach, Meth. Func. Anal. and Topol. 1 (1995), 61-85.

[25] E. W. Lytvynov, A note on test and generalized functionals of Poisson white noise, Hiroshima Math. J. 28 (1998), 463-480.

[26] E. W. Lytvynov, A. L. Rebenko, and G. V. Shchepan'uk, Wick calculus on spaces of generalized functions of compound Poisson white noise, Rep. Math. Phys. 39 (1997), 219-248.

[27] E. W. Lytvynov and G. F. Us, Dual Appell systems in non-Gaussian white noise calculus, Meth. Func. Anal. and Topol. 2 (1996), 70-85.

[28] E. B. McBride, "Obtaining Generating Functions," Springer, Berlin, New York, 1971.

[29] J. Meixner, Orthogonale Polynoksysteme mit einem besonderen Gestalt der erzeugenden Fuktion, J. London Math. Soc. 9 (1934), 6-13.

[30] P.A. Meyer, "Quantum Probability for probabilists," Lect. Notes in Math., Vol. 1538, Springer, Berlin, New York, 1993.

[31] D. Nualart and J. Vives, A duality formula on the Poisson space and some applications, in "Seminar on Stochastic Analysis, Random Fields and Applications," Vol. 36, pp. 205-213, Birkhaüser, Basel, 1995.

[32] L. Takács, "Combinatorical Methods in the Theory of Stochastic Processes," John Wiley \& Sons, New York, London, Sydney, 1967.

[33] N. Tsilevich and A. Vershik, Quasi-invariance of the gamma process and multiplicative properties of the Poisson-Dirichlet measures, C. R. Acad Sci. Paris 329 (1999), Série I, 163-168.

[34] A. M. Vershik, I. M. Gelfand, and M. I. Graev, A commutative model of representation of the group of flows $S L(2, \mathbb{R})^{X}$ that is connected with a unipotent subgroup, Funct. Anal. Appl. 17 (1983), 137-139.

[35] A. M. Vershik, I. M. Gelfand, and M. I. Graev, Models of representations of current groups, in "Representations of Lie Groups and Lie Algebras," pp. 121179, A. A. Kirilov (Ed.), Akadémiai Kiadó, Budapest, 1985. 